\title{
Top-100 Most-cited Articles on Esophageal Cancer: A Bibliometric Analysis
}

\author{
Wenjie Ni \\ Capital Medical University Affiliated Beijing Shijitan Hospital \\ Li-li Sun \\ The First People's Hospital of Yueyang \\ Tong Fang ( $\square$ fangtong653@bjsjth.cn ) \\ Capital Medical University Affiliated Beijing Shijitan Hospital
}

\section{Research}

Keywords: esophageal cancer, esophagogastric junction cancer, citation classics, bibliometric analysis, research trends

Posted Date: March 26th, 2021

DOI: https://doi.org/10.21203/rs.3.rs-362326/v1

License: (c) (i) This work is licensed under a Creative Commons Attribution 4.0 International License. Read Full License 


\section{Abstract}

Background A citation classic is a highly cited work in a field and regarded as an influential contribution to the field's advancements and literature. Analyzing citation classics and top articles promotes the recognition of research trends within a field. We present the results of the first analysis to identify the 100 most frequently cited research studies on esophageal cancer or esophagogastric junction cancer using the bibliometric analysis method.

Method We searched the Web of Science on September 24, 2020. Articles were listed in descending order by the total number of citations, and the top100 most-cited original articles on esophageal cancer or esophagogastric junction cancer were extracted and evaluated.

Results The top-100 citation classics in esophageal cancer were published from 1981 to 2018 . A significant increase was found in the number of citation classics from the early 1990s to the late 2000s, which was paralleled by an increase in randomized controlled trials focusing on the clinical treatment of tumors. The medians of the total and annual citations in our analysis were 444.50 (interquartile range [IQR] 346.25-684.50) and 30.08 (IQR 19.10-56.60), respectively. The majority of articles were published in the Journal of Clinical Oncology $(n=26)$, originated in the United States ( $n=38)$ and focused on clinical therapies $(n=59)$. The median impact factor of the journals was 27.603 (IQR 9.727-32.956).

Conclusion Our analysis provides a historical perspective on the scientific progress of esophageal cancer and contributes to the recognition of important advances in this specialty.

\section{Background}

Esophageal cancer is the eighth most common cancer and the sixth cause of death by cancer worldwide according to the Global Cancer Observatory $2018^{[1]}$. The incidence of esophageal cancer is calculated by geographical distribution. Southeast Asia, especially China, is a high incidence area, with an estimated 477,900 new cases and 375,000 deaths in 2015 alone ${ }^{[2]}$. Due to the anatomic location of the esophagus, its lymphatic drainage ranges from the lower neck to the abdominal trunk, and laterally along the esophageal wall to the surrounding trachea, heart, large vessels and vertebral body. Therefore, its recurrence and metastasis rates are very high and the overall survival is poor. A large number of research studies have been conducted in recent years with the intent to improve the overall survival rate of esophageal cancer. With the development of radiation technology and new drugs, the multidisciplinary comprehensive treatment of this cancer has gradually matured.

Retrospective analyses of historical trends in research on esophageal cancer and the examination of the literature that has had a significant impact on the field can provide insight into scientific progress over time and identify areas of greatest interest along with understudied areas. Frequently cited works often represent landmarks in the literature that have gained recognition by researchers in the field and laid the groundwork for important clinical research findings ${ }^{[3]}$. Bibliometric analyses of a discipline's most-cited articles, also called citation classics ${ }^{[4]}$, provide insight into research areas that have influenced the discipline. The number of citations and citation density (i.e., annual number of citations since the date of publication) can be used as an indicator of the influence of a certain publication on a respective field ${ }^{[5]}$. Examining citation classics in a field facilitates the identification of important literature and provides insight into research trends over time. Although articles on citation classics in the areas of critical care medicine ${ }^{[6]}$, pituitary adenoma ${ }^{[7]}$, neuroimaging ${ }^{[8]}$, neuro-oncology ${ }^{[9]}$, ischemic stroke ${ }^{[10]}$, obstetrics and gynecology ${ }^{[11]}$ and urogynecology ${ }^{[12]}$ have recently been conducted using bibliometric analysis, no studies have examined the research literature on esophageal cancer. Therefore, this study aimed to analyze the 100 most-cited original articles in the field of esophageal cancer using the bibliometric analysis method and to provide guidance to investigators in this field.

\section{Materials And Methods}

\section{Search Strategies and Inclusion Criteria}

Web of Science Core Collection includes the Science Citation Index Expanded as well as other citation indexes. We searched the Web of Science Core Collection (Clarivate Analytics) on September 24, 2020 using the following terms: [(esophageal cancer) OR (esophagogastric junction cancer) OR (esophageal carcinoma) OR (esophagogastric junction carcinoma) OR (esophagus tumor)].Incites Journal Citation Reports (JCR) was used to identify the journal impact factors.

Search results were sorted by the total number of citations in descending order. Articles were first screened by title for their relevance to the esophagus, and the remaining articles underwent a review of the abstract or the full text. Only original articles were included. Systematic reviews, secondary analyses (e.g., meta-analyses), case reports or case series, meeting reports and guidelines and consensus statements were excluded from the study because they did not reflect trends in esophageal cancer research. After two independent authors screened the studies, the top-100 most-cited articles were included in the final analysis.

Approval from an ethics committee to conduct this research was not required because this study did not involve the collection of original data from humans or animals or interventions. 
The following parameters were extracted from each article: article title, country of origin, year of publication, journal name, journal impact factor (IF; Data were collected from Incites Journal Citation Reports for the year 2019), total number of citations, number of annual citations, tumor type, study design, topic theme and type of treatment. Country of origin was based on the affiliation of the corresponding author. If there were multi-corresponding authors in an article, the first corresponding author's country was collected. The number of annual citations was calculated by dividing the total number of citations by the number of years, which was from the date of publication to 2018. Study designs included the randomized controlled trial (RCT), prospective study, retrospective study, laboratory investigation and observational study (i.e., cross-sectional study, case-control study or cohort study). In order to assess trends in the literature, articles were classified by their primary themes, which included therapies, pathogenesis or clinical presentation and imaging. Therapy-related articles were subdivided into surgery, chemotherapy, radiation therapy, chemotherapy combined with radiotherapy (i.e., chemoradiotherapy), new agents (i.e., immunotherapy) and metal stents.

\section{Statistics}

Data are presented using descriptive statistics. Spearman's correlations between continuous variables that were not normally distributed were analyzed using the Statistical Package for Social Sciences software version 23.0 (SPSS Inc., Chicago, IL). A p-value $<0.05$ was considered statistically significant.

\section{Results}

The 100 most-cited articles in the field of esophageal cancer or esophagogastric junction (EGJ) cancer and the number of citations are presented in Table 1. The total number of citations of the 100 articles ranged from 3,641 to 314, with a median of 444.50 total citations (interquartile range [IQR] 346.25-684.50) and a median of 30.08 annual citations (IQR 19.10-56.60). The top-3 ranked articles were clinical studies, whose themes were related to perioperative chemotherapy ${ }^{[13]}$, postoperative chemoradiotherapy for gastric or EGJ adenocarcinoma ${ }^{[14]}$ and preoperative chemoradiotherapy for esophageal squamous cell carcinoma (ESCC) or adenocarcinoma ${ }^{[15]}$.

\section{Source}

The 100 most-cited articles were published in 27 different journals (Table 2).

The Journal of Clinical Oncology $(\mathrm{n}=26)$, Annals of Surgery $(\mathrm{n}=10)$ and New England Journal of Medicine ( $\mathrm{n}=9)$ published the top-3 ranked articles. Journals were classified into five main categories. Fifty-one articles were published in oncology-specific journals, 17 articles in genera-specialty journals, 15 articles in surgery-specific journals, 8 articles in gastroenterology and hepatology-specific journals and 9 articles in various other journals. The journal impact factors ranged from 2.234 to 74.699 (median, 27.603; IQR 9.727-32.956). A moderately strong correlation was found between impact factor and total number of citations reported in a given journal (Spearman's coefficient $=0.552, p<0.001$ ), and the number of annual citations strongly correlated with the total number of citations (Spearman's coefficient $=0.715, p<0.001$ ).

The majority of the articles originated in the United States (US) with 38 publications, Japan and Germany, both with 15 publications and the United Kingdom with 9 articles, which was ranked third (Figure 1).

\section{Study Design and Themes}

The most frequent study design was the RCT $(n=42)$, followed by the prospective trial $(n=17)$, retrospective study $(n=17)$ and laboratory investigation $(n=15)$. Observational studies accounted for only $9 \%(9 / 100)$ of all studies.

The most common themes were therapies $(n=59)$, followed by pathogenesis or clinical presentation $(n=34)$ and imaging $(n=7)$. Among the therapeutic trials, 22 pertained to chemotherapy, 19 to chemoradiotherapy and 14 to surgery (Figure 2).

\section{Therapies}

The citations were categorized into five types of treatment based on the treatment's purpose, which represented the main areas of research in esophageal cancer: (1) definitive treatment $(n=20),(2)$ perioperative treatment $(n=3),(3)$ preoperative treatment $(n=19)$, (4) postoperative treatment $(n=2)$ and (5) salvage treatment $(n=15)$. 
The largest category was radical treatment (20 articles). Six articles on the use of radical chemoradiotherapy for ESCC and/or esophageal adenocarcinoma were published in 1997-2007. Among these, one study published in 1999 explored the T4 and/or M1 lymph node in ESCC. Three citation classics, the RTOG 85-01 (two articles published) and RTOG 94-05 studies, reported the effect of concurrent chemoradiotherapy for locally advanced esophageal cancer (1997-2002). Two studies examined chemoradiotherapy with/without surgery in patients with ESCC (2005-2007) and the remaining 14 articles were related to surgery (1991-2012). Two articles were published in 1991 and 1995 on surgical approaches for esophageal cancer in Japan (i.e., three-field versus two-field lymphadenectomy). Five studies focused on the prognostic factors after surgery for esophageal cancer, including the classification of EGJ, surgical technique, histologic tumor type, tumor length, lymph node status and the number of lymph nodes removed (2000-2008). Four studies focused on the very early stage of esophageal cancer (2005-2008) and three articles pertained to minimally invasive esophagectomy (2006-2012). Three articles were about perioperative chemotherapy versus surgery alone for patients with locally advanced adenocarcinoma of the esophagus, EGJ and gastric cancers and ESCC (1988-2011).

Only two articles pertained to postoperative therapy. Macdonald et al. reported advantages of adjuvant chemoradiotherapy for adenocarcinoma of the esophagus or gastric cancer, and Ando et al. studied the value of postoperative chemotherapy for ESCC (2001-2003).

Seven studies explored optimal salvage chemotherapy regimens for advanced gastric cancer and/or esophageal cancer, especially adenocarcinoma (1997-2014). The article (known as the ToGA study), on the use of trastuzumab combined with chemotherapy for the treatment of HER2-positive advanced gastric or EGJ cancer, was published in 2010. The article (known as the REAL-3 study) on the use of panitumumab, an epidermal growthfactor receptor (EGFR) inhibitor, combined with chemotherapy in patients with EGFR positive advanced esophagogastric cancer, was published in 2013. Three articles assessed the value of the vascular EGFR inhibitor in patients with advanced gastric cancer during 2014-2016. The use of immunotherapy for advanced gastric and EGJ cancers has increased in recent years, producing two recent articles. One 1993 study reported the use of metal stents for palliation of esophageal obstruction.

Nineteen citations pertained to preoperative treatment. Among these, 12 articles reviewed the use of neoadjuvant concurrent chemoradiotherapy for esophageal cancer, 6 articles were about preoperative chemotherapy and 1 examined the value of preoperative radiotherapy.

Pathogenesis or Clinical Presentation

A total of 34 citations focused on tumor pathogenesis or clinical presentation. Among these, 16 reported clinical or pathological characteristics of esophageal cancer. Among the 18 pathogenesis-related studies, 9 focused on the etiology of exogenous factors, such as obesity, alcohol intake, tobacco smoking, nutrition deficiencies, biotoxins, medicines and gastroesophageal reflux. The remaining 9 articles were related to gene levels, such as p53, microRNA, SOX2, MTS1/CDK4I and PLCE1.

Imaging

Seven articles pertained to imaging (2000-2011). Among these, 5 explored the predictors of response to chemotherapy or chemoradiotherapy among patients with esophageal cancer using positive emission tomography. One study investigated the utility of positive emission tomography for the staging of patients with potentially operable esophageal carcinoma. The remaining study focused on the early detection of superficial squamous cell carcinoma in the head and neck regions and esophagus using narrow band imaging.

Temporal Trends

There has been a sharp increase in the number of cited articles since the early 1990s. The peak period with the largest number of cited articles was 2005-2008 ( $n=23)$, with a steep decline afterwards (Figure 3A). This increase was largely attributable to studies pertaining to therapies (Figure 3B), especially those focusing on surgery, chemoradiotherapy and chemotherapy (Figure 3C). Furthermore, immunotherapy, which was a promising treatment was developing rapidly and produced 2 of the most-cited articles within two years. Since the 1990s, RCTs have increased continuously and steadily. Prospective studies have also increased gradually with a decrease in retrospective studies, which finally reached its peak during $2005-2008$ (Figure 3D).

\section{Discussion}

Citation analysis can be a useful tool for identifying publications that have a high scientific impact. A bibliometric analysis of the most-cited articles highlights the most influential articles, trending topics and prolific institutions that contribute to the evolution of a scientific subspecialty. Our study depicts the top 100 citation classics that have played a significant role in bolstering progress in the field of esophageal cancer.

In our analysis, the types of pathology found in the patients in most of the studies were adenocarcinoma with or without squamous cell carcinoma, while some of the other studies involving esophageal squamous cell carcinoma were from Japan and a few were from Germany and France. This

Page $4 / 25$ 
finding also reflects the characteristics of the distribution of esophageal cancer. The research mostly pertained to clinical treatment, followed by pathogenesis or clinical presentation. With developments in the field of medicine, the numbers of RCTs and prospective studies of multidisciplinary treatments are booming, thereby providing more evidence-based rationales for clinicians' treatment decisions.

Analyses of the citation classics showed that more than half of the 100 most-cited articles were published in oncology-specific journals, and $45 \%$ of the articles were published in journals with very high impact factors, including Japan Clinical Onclogy, Annals of Surgery and the New England Journal of Medicine. Most of the remaining articles were published in journals with a relatively low impact factor. Thus, the median impact factor was 27.603. Although the articles published in top journals are cited more frequently, the articles published in top journals, such as the New England Journal of Medicine are relatively rare. Therefore, the correlation between the impact factor and total number of citations of classic papers in this study was not strong. However, a strong correlation was found between the annual and total numbers of citations, indicating the influence of the research reported in the classic papers in the field is persistent and has not been diluted by time.

Most of the classic citations were from the US, which is consistent with the results of most other cancer-related studies. This likely reflects a bias of US authors to submit studies for publication in US-based journals and of US reviewers toward articles from authors and studies based in the US ${ }^{[16]}$. Of note, we did not identify articles in languages other than English; thus, we have not represented the entire body of literature that could possibly be relevant to the field of esophageal cancer. That said, this same bias toward English-language articles, made clear by past research revealing that non-Englishlanguage articles have low acceptace and citation rates, reduces the likelihood of increasing the number of citations in non-English-language articles ${ }^{[17}$, 18].

The most-cited articles focused mostly on optimal clinical treatment in RCTs along with therapeutic studies. Among these, the classic articles on radical chemoradiotherapy were the RTOG $85-01^{[19,20]}$ and RTOG $94-05$ studies, ${ }^{[21]}$ which established guidelines for locally advanced esophageal cancer. The studies from Japan revealed that three-field lymphadenectomy has better local control and overall survival than two-field lymphadenectomy[22-25]. However, three-field lymphadenectomy could only be performed in a hospital; thus, it has not been adopted worldwide. Furthermore, the persistence and the recurrence rate of esophageal cancer were high $(24-58 \%[19-21,26-30])$, and the 5 -year overall survival was low $\left(5-25 \%{ }^{[31]}\right)$ after radical chemoradiotherapy or surgery alone. This finding has promoted multidisciplinary treatment studies, which include surgery combined with chemotherapy or chemoradiotherapy ${ }^{[32-37]}$. Due to the backwardness of radiotherapy technology and the high incidence of surgical complications in earlier years, most of the prospective preoperative chemoradiotherapy studies had insufficient sample sizes or controversial results. In 2012 , a Dutch study (known as the CROSS study) confirmed that the use of neoadjuvant chemoradiotherapy for esophageal cancer has better overall survival and disease free survival than surgery alone, which had been recommended as standard treatment for those with locally advanced esophageal cancer ${ }^{[15]}$. The long-term follow-up results further confirmed the value of preoperative chemoradiotherapy ${ }^{[38]}$. Since then, neoadjuvant chemoradiotherapy for esophageal cancer has entered a new era.

Three citation classics in perioperative chemotherapy mainly focused on adenocarcinoma of the esophagus or stomach ${ }^{[13,39,40]}$, which might not have been suitable for esophageal squamous cell carcinoma. Similarly, the RCT of postoperative chemoradiotherapy also focused on adenocarcinoma of the esophagus or stomach ${ }^{[14]}$. Postoperative chemotherapy only improved the disease free survival for patients with ESCC ${ }^{[41]}$. Based on the citation classics, no recommendations have been made for adjuvant therapy after radical esophagectomy for ESCC.

Salvage treatment studies have gradually changed from chemotherapy ${ }^{[42-44]}$ to targeted therapy ${ }^{[45-48]}$, with the discovery of molecular checkpoints. Of note, immunotherapy has also attracted much attention in recent years, opening a new door to the treatment of esophageal cancer. At present, the role of immunotherapy in neoadjuvant, adjuvant or radical therapy is still unclear, and many studies are ongoing.

Although bibliometric analysis can provide a comprehensive understanding of the important studies on esophageal cancer, citation analysis has drawn criticism owing to the effects of time ${ }^{[16,49,50]}$. Some hypothesize that older studies have more time to accumulate citations, making them more likely to end up on the list of citation classics. Articles that have become "landmark" studies may be cited less often over time as their conclusions become more commonplace, a phenomenon referred to as "obliteration by incorporation" ${ }^{16,50]}$. To mitigate the effect of time in this way, the number of annual citations was introduced.

However, the time lag between publication and accumulation of sufficient citations for incorporation into a list of citation classics, such as our top-100 classics, must be acknowledged, and one cannot predict which of the more recent articles will rise to be included as a citation classic and within what time frame. For this reason, fields must periodically update their list of classics in keeping with this trend.

\section{Abbreviations}

EGFR, epidermal growth-factor receptor

ESCC, esophageal squamous cell carcinoma

EGJ, esophagogastric junction

HER2, human epidermal growth factor receptor 2 
$\mathrm{RCT}$, randomized controlled trial

US, United States

\section{Declarations}

Ethics approval and consent to participate: Not applicable

Consent for publication: Not applicable

Availability of data and materials: Not applicable

Competing interests: The authors declare that they have no competing interests

Funding: Not applicable

Authors' contributions: NWJ analyzed the data and wrote the article. NWJ and SLL collected the data. FT made substantial contributions to the conception and design of the study, revised the article and gave final approval of the version to be published. All authors read and approved the final manuscript.

\section{Tables}

Table 1. The 100 most-cited articles on esophageal cancer ranked in order of the number of citations received. 


\begin{tabular}{|c|c|c|c|c|c|c|c|c|}
\hline Rank & Article & Country & $\begin{array}{l}\text { Total } \\
\text { number } \\
\text { of } \\
\text { citations }\end{array}$ & $\begin{array}{l}\text { Number } \\
\text { of } \\
\text { annual } \\
\text { citations }\end{array}$ & $\begin{array}{l}\text { Tumor } \\
\text { type }\end{array}$ & Study design & Theme & Treatment \\
\hline 1 & $\begin{array}{l}\text { Perioperative } \\
\text { chemotherapy versus } \\
\text { surgery alone for } \\
\text { resectable } \\
\text { gastroesophageal } \\
\text { cancer. New England } \\
\text { Journal of Medicine } \\
2006 ; 355 \text { : } 11-20 \text {. }\end{array}$ & UK & 3,641 & 260.07 & $A E G+G$ & $\mathrm{RCT}$ & Chemotherapy & Perioperative \\
\hline 2 & $\begin{array}{l}\text { Chemoradiotherapy } \\
\text { after surgery compared } \\
\text { with surgery alone for } \\
\text { adenocarcinoma of the } \\
\text { stomach or } \\
\text { gastroesophageal } \\
\text { junction. New England } \\
\text { Journal of Medicine } \\
\text { 2001; 345: 725-730. }\end{array}$ & USA & 2,513 & 132.26 & $A E G+G$ & RCT & Chemoradiotherapy & Postoperative \\
\hline 3 & $\begin{array}{l}\text { Preoperative } \\
\text { Chemoradiotherapy for } \\
\text { Esophageal or } \\
\text { Junctional Cancer. New } \\
\text { England Journal of } \\
\text { Medicine 2012; 366: } \\
\text { 2074-2084. }\end{array}$ & Netherlands & 2,351 & 293.88 & Ade+ESCC & $\mathrm{RCT}$ & Chemoradiotherapy & Preoperative \\
\hline 4 & $\begin{array}{l}\text { Symptomatic } \\
\text { gastroesophageal reflux } \\
\text { as a risk factor for } \\
\text { esophageal } \\
\text { adenocarcinoma. New } \\
\text { England Journal of } \\
\text { Medicine 1999; 340: } \\
\text { 825-831. }\end{array}$ & Sweden & 2,129 & 101.38 & $A E G+G$ & $\begin{array}{l}\text { Case-control } \\
\text { study }\end{array}$ & $\begin{array}{l}\text { Pathogenesis/Clinical } \\
\text { presentation }\end{array}$ & - \\
\hline 5 & $\begin{array}{l}\text { Rising incidence of } \\
\text { adenocarcinoma of the } \\
\text { esophagus and gastric } \\
\text { cardia. Jama 1991; } 265 \text { : } \\
\text { 1287-1289. }\end{array}$ & USA & 2,005 & 69.14 & $A E G+G$ & $\begin{array}{l}\text { Cross- } \\
\text { sectional } \\
\text { study }\end{array}$ & $\begin{array}{l}\text { Pathogenesis/Clinical } \\
\text { presentation }\end{array}$ & - \\
\hline 6 & $\begin{array}{l}\text { Capecitabine and } \\
\text { oxaliplatin for advanced } \\
\text { esophagogastric cancer. } \\
\text { New England Journal of } \\
\text { Medicine 2008; 358: 36- } \\
46 .\end{array}$ & UK & 1,537 & 128.08 & $\mathrm{AEG}+\mathrm{G}$ & $\mathrm{RCT}$ & Chemotherapy & Salvage \\
\hline 7 & $\begin{array}{l}\text { A comparison of } \\
\text { multimodal therapy and } \\
\text { surgery for esophageal } \\
\text { adenocarcinoma. New } \\
\text { England Journal of } \\
\text { Medicine 1996; 335: } \\
\text { 462-467. }\end{array}$ & Ireland & 1,523 & 63.46 & $\mathrm{AE}$ & RCT & Chemoradiotherapy & Preoperative \\
\hline 8 & $\begin{array}{l}\text { Chemoradiotherapy of } \\
\text { locally advanced } \\
\text { esophageal cancer - } \\
\text { Long-term follow-up of } \\
\text { a prospective } \\
\text { randomized trial (RTOG } \\
85-01) \text {. Jama-Journal of } \\
\text { the American Medical } \\
\text { Association 1999; } 281 \text { : } \\
\text { 1623-1627. }\end{array}$ & USA & 1,319 & 62.81 & Ade+ESCC & $\mathrm{RCT}$ & Chemoradiotherapy & $\begin{array}{l}\text { Radical } \\
\text { treatment }\end{array}$ \\
\hline 9 & $\begin{array}{l}\text { Pathologic assessment } \\
\text { of tumor regression } \\
\text { after preoperative } \\
\text { chemoradiotherapy of } \\
\text { esophageal carcinoma. } \\
\text { Clinicopathologic } \\
\text { correlations. Cancer } \\
\text { 1994; } 73 \text { : } 2680-2686 .\end{array}$ & France & 1,247 & 47.96 & ESCC & $\begin{array}{l}\text { Retrospective } \\
\text { study }\end{array}$ & Chemoradiotherapy & - \\
\hline 10 & $\begin{array}{l}\text { Ramucirumab } \\
\text { monotherapy for } \\
\text { previously treated }\end{array}$ & USA & 1,173 & 195.50 & $A E G+G$ & RCT & Chemotherapy & Salvage \\
\hline
\end{tabular}


advanced gastric or

gastro-oesophageal

junction

adenocarcinoma

(REGARD): an

international,

randomised,

multicentre, placebo-

controlled, phase 3 trial.

Lancet 2014; 383: 31-

39.

\begin{tabular}{|c|c|c|c|c|c|c|c|c|}
\hline 11 & $\begin{array}{l}\text { Ramucirumab plus } \\
\text { paclitaxel versus } \\
\text { placebo plus paclitaxel } \\
\text { in patients with } \\
\text { previously treated } \\
\text { advanced gastric or } \\
\text { gastro-oesophageal } \\
\text { junction } \\
\text { adenocarcinoma } \\
\text { (RAINBOW): a double- } \\
\text { blind, randomised } \\
\text { phase } 3 \text { trial. Lancet } \\
\text { Oncology 2014; 15: } \\
\text { 1224-1235. }\end{array}$ & Germany & 1,095 & 182.50 & $A E G+G$ & $\mathrm{RCT}$ & Chemotherapy & Salvage \\
\hline 12 & $\begin{array}{l}\text { Chemoradiotherapy } \\
\text { followed by surgery } \\
\text { compared with surgery } \\
\text { alone in squamous-cell } \\
\text { cancer of the } \\
\text { esophagus. New } \\
\text { England Journal of } \\
\text { Medicine 1997; 337: } \\
\text { 161-167. }\end{array}$ & France & 1,049 & 45.61 & ESCC & $\mathrm{RCT}$ & Chemoradiotherapy & Preoperative \\
\hline 13 & $\begin{array}{l}\text { Perioperative } \\
\text { Chemotherapy } \\
\text { Compared With Surgery } \\
\text { Alone for Resectable } \\
\text { Gastroesophageal } \\
\text { Adenocarcinoma: An } \\
\text { FNCLCC and FFCD } \\
\text { Multicenter Phase III } \\
\text { Trial. Journal of Clinical } \\
\text { Oncology 2011; } 29 \text { : } \\
\text { 1715-1721. }\end{array}$ & France & 1,043 & 115.89 & $\mathrm{AE}$ & $\mathrm{RCT}$ & Chemotherapy & Perioperative \\
\hline 14 & $\begin{array}{l}\text { Surgical resection with } \\
\text { or without preoperative } \\
\text { chemotherapy in } \\
\text { oesophageal cancer: a } \\
\text { randomised controlled } \\
\text { trial. Lancet 2002; 359: } \\
\text { 1727-1733. }\end{array}$ & UK & 1,024 & 56.89 & Ade+ESCC & $\mathrm{RCT}$ & Chemotherapy & Preoperative \\
\hline 15 & $\begin{array}{l}\text { INT } 0123 \text { (radiation } \\
\text { therapy oncology group } \\
94-05 \text { ) phase III trial of } \\
\text { combined-modality } \\
\text { therapy for esophageal } \\
\text { cancer: High-dose } \\
\text { versus standard-dose } \\
\text { radiation therapy. } \\
\text { Journal of Clinical } \\
\text { Oncology 2002; } 20 \text { : } \\
\text { 1167-1174. }\end{array}$ & USA & 1,017 & 56.50 & Ade+ESCC & $\mathrm{RCT}$ & Chemoradiotherapy & $\begin{array}{l}\text { Radical } \\
\text { treatment }\end{array}$ \\
\hline 16 & $\begin{array}{l}\text { Chemotherapy followed } \\
\text { by surgery compared } \\
\text { with surgery alone for } \\
\text { localized esophageal } \\
\text { cancer. New England } \\
\text { Journal of Medicine } \\
\text { 1998; 339: 1979-1984. }\end{array}$ & USA & 993 & 45.14 & Ade+ESCC & $\mathrm{RCT}$ & Chemotherapy & Preoperative \\
\hline 17 & $\begin{array}{l}\text { Randomized trial of } \\
\text { preoperative } \\
\text { chemoradiation versus } \\
\text { surgery alone in } \\
\text { patients with } \\
\text { locoregional } \\
\text { esophageal carcinoma. } \\
\text { Journal of Clinical }\end{array}$ & USA & 988 & 52.00 & Ade+ESCC & $\mathrm{RCT}$ & Chemoradiotherapy & Preoperative \\
\hline
\end{tabular}


Oncology 2001; 19: 305-

313.

\begin{tabular}{|c|c|c|c|c|c|c|c|c|}
\hline 18 & $\begin{array}{l}\text { The role of } \\
\text { overdiagnosis and } \\
\text { reclassification in the } \\
\text { marked increase of } \\
\text { esophageal } \\
\text { adenocarcinoma } \\
\text { incidence. Journal of } \\
\text { the National Cancer } \\
\text { Institute 2005; } 97: 142- \\
146 .\end{array}$ & USA & 934 & 62.27 & $\mathrm{AE}$ & $\begin{array}{l}\text { Cross- } \\
\text { sectional } \\
\text { study }\end{array}$ & $\begin{array}{l}\text { Pathogenesis/Clinical } \\
\text { presentation }\end{array}$ & - \\
\hline 19 & $\begin{array}{l}\text { Phase III trial of } \\
\text { trimodality therapy with } \\
\text { cisplatin, fluorouracil, } \\
\text { radiotherapy, and } \\
\text { surgery compared with } \\
\text { surgery alone for } \\
\text { esophageal cancer: } \\
\text { CALGB 9781. Journal of } \\
\text { Clinical Oncology } 2008 \text {; } \\
26: 1086-1092 \text {. }\end{array}$ & USA & 881 & 73.42 & Ade+ESCC & $\mathrm{RCT}$ & Chemoradiotherapy & Preoperative \\
\hline 20 & $\begin{array}{l}\text { Chemoradiation with } \\
\text { and without surgery in } \\
\text { patients with locally } \\
\text { advanced squamous } \\
\text { cell carcinoma of the } \\
\text { esophagus. Journal of } \\
\text { Clinical Oncology 2005; } \\
\text { 23: } 2310-2317 \text {. }\end{array}$ & Germany & 845 & 56.33 & ESCC & RCT & Chemoradiotherapy & $\begin{array}{l}\text { Radical } \\
\text { treatment }\end{array}$ \\
\hline 21 & $\begin{array}{l}\text { Minimally invasive } \\
\text { versus open } \\
\text { oesophagectomy for } \\
\text { patients with } \\
\text { oesophageal cancer: a } \\
\text { multicentre, open-label, } \\
\text { randomised controlled } \\
\text { trial. Lancet 2012; 379: } \\
\text { 1887-1892. }\end{array}$ & Netherlands & 811 & 101.38 & Ade+ESCC & $\mathrm{RCT}$ & Surgery & $\begin{array}{l}\text { Radical } \\
\text { treatment }\end{array}$ \\
\hline 22 & $\begin{array}{l}\text { Neoadjuvant } \\
\text { chemoradiotherapy plus } \\
\text { surgery versus surgery } \\
\text { alone for oesophageal } \\
\text { or junctional cancer } \\
\text { (CROSS): Iong-term } \\
\text { results of a randomised } \\
\text { controlled trial. Lancet } \\
\text { Oncology 2015; 16: } \\
\text { 1090-1098. }\end{array}$ & Netherlands & 802 & 160.40 & Ade+ESCC & $\mathrm{RCT}$ & Chemoradiotherapy & Preoperative \\
\hline 23 & $\begin{array}{l}\text { Chemoradiation } \\
\text { followed by surgery } \\
\text { compared with } \\
\text { chemoradiation alone in } \\
\text { squamous cancer of the } \\
\text { esophagus: FFCD } 9102 \text {. } \\
\text { Journal of Clinical } \\
\text { Oncology 2007; } 25 \text { : } \\
1160-1168 \text {. }\end{array}$ & France & 772 & 59.38 & ESCC & RCT & Chemoradiotherapy & $\begin{array}{l}\text { Radical } \\
\text { treatment }\end{array}$ \\
\hline 24 & $\begin{array}{l}\text { Randomized trial } \\
\text { comparing epirubicin, } \\
\text { cisplatin, and } \\
\text { fluorouracil versus } \\
\text { fluorouracil, } \\
\text { doxorubicin, and } \\
\text { methotrexate in } \\
\text { advanced } \\
\text { esophagogastric cancer. } \\
\text { Journal of Clinical } \\
\text { Oncology 1997; } 15: 261 \text { - } \\
267 \text {. }\end{array}$ & UK & 747 & 32.48 & $\mathrm{AE}$ & RCT & Chemotherapy & Salvage \\
\hline 25 & $\begin{array}{l}\text { Surgery alone versus } \\
\text { chemoradiotherapy } \\
\text { followed by surgery for } \\
\text { resectable cancer of the } \\
\text { oesophagus: a } \\
\text { randomised controlled } \\
\text { phase III trial. Lancet }\end{array}$ & Australia & 698 & 46.53 & Ade+ESCC & RCT & Chemoradiotherapy & Preoperative \\
\hline
\end{tabular}


Oncology 2005; 6: 659-

668.

soX2 is an amplified

USA

644

58.55

ESCC

Laboratory

investigation

Pathogenesis/Clinical presentation

oncogene in lung and

esophageal squamous

cell carcinomas. Nature

Genetics 2009; 41:

1238-U1105.

$27 \begin{aligned} & \text { A controlled trial of an } \\ & \text { expansile metal stent } \\ & \text { for palliation of } \\ & \text { esophageal obstruction } \\ & \text { due to inoperable } \\ & \text { cancer. New England } \\ & \text { Journal of Medicine } \\ & \text { 1993; 329: 1302-1307. }\end{aligned}$

Phase III Comparison of

Germany

635

23.52

Ade+ESCC RCT

Metal stent

Salvage

for palliation of

due to inoperable

cancer. New England

ournal of Medicine

Preoperative

Chemotherapy

Compared With

Chemoradiotherapy in

Patients With Locally

Advanced

Adenocarcinoma of the

Esophagogastric

Junction. Journal of

Clinical Oncology 2009;

27: 851-856.

29 A Randomized Trial

Japan

585

73.13

ESCC

RCT

Chemotherapy

Preoperative

Postoperative Adjuvant

Chemotherapy with

Cisplatin and 5-

Fluorouracil Versus

Preoperative

Chemotherapy for

Localized Advanced

Squamous Cell

Carcinoma of the

Thoracic Esophagus

(JCOG9907). Annals of

Surgical Oncology 2012;

19: 68-74.

\begin{tabular}{|c|c|c|c|c|c|c|c|c|}
\hline 30 & $\begin{array}{l}\text { Long-Term Results of a } \\
\text { Randomized Trial of } \\
\text { Surgery With or Without } \\
\text { Preoperative } \\
\text { Chemotherapy in } \\
\text { Esophageal Cancer. } \\
\text { Journal of Clinical } \\
\text { Oncology } 2009 ; 27 \text { : } \\
5062-5067 \text {. }\end{array}$ & UK & 581 & 52.82 & Ade+ESCC & $\mathrm{RCT}$ & Chemotherapy & Preoperative \\
\hline 31 & $\begin{array}{l}\text { Nivolumab in patients } \\
\text { with advanced gastric } \\
\text { or gastro-oesophageal } \\
\text { junction cancer } \\
\text { refractory to, or } \\
\text { intolerant of, at least } \\
\text { two previous } \\
\text { chemotherapy regimens } \\
\text { (ONO-4538-12, } \\
\text { ATTRACTION-2): a } \\
\text { randomised, double- } \\
\text { blind, placebo- } \\
\text { controlled, phase } 3 \text { trial. } \\
\text { Lancet 2017; } 390: 2461- \\
2471 \text {. }\end{array}$ & Japan & 562 & 187.33 & $A E G+G$ & RCT & $\begin{array}{l}\text { New } \\
\text { agents_immunotherapy }\end{array}$ & Salvage \\
\hline 32 & $\begin{array}{l}\text { PET to assess early } \\
\text { metabolic response and } \\
\text { to guide treatment of } \\
\text { adenocarcinoma of the } \\
\text { oesophagogastric } \\
\text { junction: The MUNICON } \\
\text { phase II trial. Lancet } \\
\text { Oncology 2007; 8: 797- } \\
805 \text {. }\end{array}$ & Germany & 551 & 42.38 & $\mathrm{AE}$ & $\begin{array}{l}\text { Prospective } \\
\text { study }\end{array}$ & Imaging & - \\
\hline 33 & Prediction of response & Germany & 545 & 28.68 & $\mathrm{AE}$ & Prospective & Imaging & - \\
\hline
\end{tabular}

Page 10/25 


\begin{tabular}{|c|c|c|c|c|c|c|c|c|}
\hline & $\begin{array}{l}\text { to preoperative } \\
\text { chemotherapy in } \\
\text { adenocarcinomas of the } \\
\text { esophagogastric } \\
\text { junction by metabolic } \\
\text { imaging. Journal of } \\
\text { Clinical Oncology 2001; } \\
\text { 19: } 3058-3065 \text {. }\end{array}$ & & & & & study & & \\
\hline 34 & $\begin{array}{l}\text { Clinical significance of } \\
\text { programmed death-1 } \\
\text { ligand-1 and } \\
\text { programmed death-1 } \\
\text { ligand-2 expression in } \\
\text { human esophageal } \\
\text { cancer. Clinical Cancer } \\
\text { Research 2005; } 11 \text { : } \\
\text { 2947-2953. }\end{array}$ & Japan & 541 & 36.07 & ESCC & $\begin{array}{l}\text { Laboratory } \\
\text { investigation }\end{array}$ & $\begin{array}{l}\text { Pathogenesis/Clinical } \\
\text { presentation }\end{array}$ & - \\
\hline 35 & $\begin{array}{l}\text { Progress report of } \\
\text { combined } \\
\text { chemoradiotherapy } \\
\text { versus radiotherapy } \\
\text { alone in patients with } \\
\text { esophageal cancer: An } \\
\text { intergroup study. } \\
\text { Journal of Clinical } \\
\text { Oncology 1997; } 15: 277- \\
284 \text {. }\end{array}$ & USA & 536 & 23.30 & Ade+ESCC & RCT & Chemoradiotherapy & $\begin{array}{l}\text { Radical } \\
\text { treatment }\end{array}$ \\
\hline 36 & $\begin{array}{l}\text { Phase III trial in } \\
\text { metastatic } \\
\text { gastroesophageal } \\
\text { adenocarcinoma with } \\
\text { fluorouracil, leucovorin } \\
\text { plus either oxaliplatin or } \\
\text { cisplatin: A study of the } \\
\text { arbeitsgemeinschaft } \\
\text { internistische onkologie. } \\
\text { Journal of Clinical } \\
\text { Oncology 2008; 26: } \\
\text { 1435-1442. }\end{array}$ & Germany & 506 & 42.17 & $A E G+G$ & RCT & Chemotherapy & Salvage \\
\hline 37 & $\begin{array}{l}\text { Extended transthoracic } \\
\text { resection compared } \\
\text { with limited transhiatal } \\
\text { resection for } \\
\text { adenocarcinoma of the } \\
\text { mid/distal esophagus - } \\
\text { Five-year survival of a } \\
\text { randomized clinical } \\
\text { trial. Annals of Surgery } \\
2007 ; 246: 992-1001 \text {. }\end{array}$ & Nethrlands & 506 & 38.92 & $\mathrm{AE}$ & RCT & Surgery & $\begin{array}{l}\text { Radical } \\
\text { treatment }\end{array}$ \\
\hline 38 & $\begin{array}{l}\text { Adenocarcinoma of the } \\
\text { esophagogastric } \\
\text { junction - Results of } \\
\text { surgical therapy based } \\
\text { on } \\
\text { anatomical/topographic } \\
\text { classification in } 1,002 \\
\text { consecutive patients. } \\
\text { Annals of Surgery } 2000 \text {; } \\
\text { 232: } 353-360 \text {. }\end{array}$ & Germany & 500 & 25.00 & $\mathrm{AE}$ & $\begin{array}{l}\text { Retrospective } \\
\text { study }\end{array}$ & Surgery & $\begin{array}{l}\text { Radical } \\
\text { treatment }\end{array}$ \\
\hline 39 & $\begin{array}{l}\text { Trastuzumab in } \\
\text { combination with } \\
\text { chemotherapy versus } \\
\text { chemotherapy alone for } \\
\text { treatment of HER2-- } \\
\text { positive advanced } \\
\text { gastric or gastro- } \\
\text { oesophageal junction } \\
\text { cancer (ToGA): a phase } \\
\text { 3, open-label, } \\
\text { randomised controlled } \\
\text { trial. Lancet 2010; } 376 \text { : } \\
687-697 \text {. }\end{array}$ & $\begin{array}{l}\text { South } \\
\text { Korea }\end{array}$ & 494 & 49.40 & $A E G+G$ & RCT & Chemotherapy & Salvage \\
\hline 40 & $\begin{array}{l}\text { An endoscopic biopsy } \\
\text { protocol can } \\
\text { differentiate high-grade } \\
\text { dysplasia from early } \\
\text { adenocarcinoma in } \\
\text { Barrett's esophagus. }\end{array}$ & USA & 480 & 17.78 & $\mathrm{AE}$ & $\begin{array}{l}\text { Prospective } \\
\text { study }\end{array}$ & $\begin{array}{l}\text { Pathogenesis/Clinical } \\
\text { presentation }\end{array}$ & - \\
\hline
\end{tabular}

Page $11 / 25$ 
Gastroenterology 1993; 105: 40-50.

$41 \quad$ Surgery plus

Japan

471

27.71

ESCC

RCT

Chemotherapy

Postoperative

chemotherapy

compared with surgery

alone for localized

squamous cell

carcinoma of the

thoracic esophagus: A

Japan Clinical Oncology

Group Study -

JCOG9204. Journal of

Clinical Oncology 2003;

21: 4592-4596.

\begin{tabular}{|c|c|c|c|c|c|c|c|c|}
\hline 42 & $\begin{array}{l}\text { Simultaneous } \\
\text { occurrence of fumonisin } \\
\text { B1 and other } \\
\text { mycotoxins in moldy } \\
\text { corn collected from the } \\
\text { People's Republic of } \\
\text { China in regions with } \\
\text { high incidences of } \\
\text { esophageal cancer. } \\
\text { Applied and } \\
\text { Environmental } \\
\text { Microbiology 1994; } 60 \text { : } \\
\text { 847-852. }\end{array}$ & USA & 470 & 18.08 & ESCC & $\begin{array}{l}\text { Laboratory } \\
\text { investigation }\end{array}$ & $\begin{array}{l}\text { Pathogenesis/Clinical } \\
\text { presentation }\end{array}$ & - \\
\hline 43 & $\begin{array}{l}\text { Pre-operative } \\
\text { radiotherapy prolongs } \\
\text { survival in operable } \\
\text { esophageal carcinoma: } \\
\text { a randomized, } \\
\text { multicenter study of pre- } \\
\text { operative radiotherapy } \\
\text { and chemotherapy. The } \\
\text { second Scandinavian } \\
\text { trial in esophageal } \\
\text { cancer. World Journal of } \\
\text { Surgery 1992; 16: 1104- } \\
1110 \text {. }\end{array}$ & Norway & 465 & 16.61 & ESCC & RCT & Radiation & Preoperative \\
\hline 44 & $\begin{array}{l}\text { Randomized, Double- } \\
\text { Blind, Placebo- } \\
\text { Controlled Phase III Trial } \\
\text { of Apatinib in Patients } \\
\text { With Chemotherapy- } \\
\text { Refractory Advanced or } \\
\text { Metastatic } \\
\text { Adenocarcinoma of the } \\
\text { Stomach or } \\
\text { Gastroesophageal } \\
\text { Junction. Journal of } \\
\text { Clinical Oncology 2016; } \\
34: 1448-1454 \text {. }\end{array}$ & China & 460 & 115.00 & $A E G+G$ & RCT & Chemotherapy & Salvage \\
\hline 45 & $\begin{array}{l}\text { Frequent somatic } \\
\text { mutation of the } \\
\text { MTS1/CDK4I (multiple } \\
\text { tumor } \\
\text { suppressor/cyclin- } \\
\text { dependent kinase } 4 \\
\text { inhibitor) gene in } \\
\text { esophageal squamous } \\
\text { cell carcinoma. Cancer } \\
\text { Research 1994; } 54 \text { : } \\
\text { 3396-3397. }\end{array}$ & Japan & 459 & 17.65 & ESCC & $\begin{array}{l}\text { Laboratory } \\
\text { investigation }\end{array}$ & $\begin{array}{l}\text { Pathogenesis/clinical } \\
\text { presentation }\end{array}$ & - \\
\hline 46 & $\begin{array}{l}\text { Epirubicin, oxaliplatin, } \\
\text { and capecitabine with } \\
\text { or without } \\
\text { panitumumab for } \\
\text { patients with previously } \\
\text { untreated advanced } \\
\text { oesophagogastric } \\
\text { cancer (REAL3): a } \\
\text { randomised, open-label } \\
\text { phase } 3 \text { trial. Lancet } \\
\text { Oncology 2013; } 14: 481 \text { - } \\
489 \text {. }\end{array}$ & UK & 458 & 65.43 & $A E G+G$ & RCT & Chemotherapy & Salvage \\
\hline 47 & $\begin{array}{l}\text { Long-term results and } \\
\text { risk factor analysis for } \\
\text { recurrence after curative }\end{array}$ & Germany & 455 & 37.92 & $\mathrm{AE}$ & $\begin{array}{l}\text { Prospective } \\
\text { study }\end{array}$ & Surgery & $\begin{array}{l}\text { Radical } \\
\text { treatment }\end{array}$ \\
\hline
\end{tabular}

Page 12/25 


\begin{tabular}{|c|c|c|c|c|c|c|c|c|}
\hline & $\begin{array}{l}\text { endoscopic therapy in } \\
349 \text { patients with high- } \\
\text { grade intraepithelial } \\
\text { neoplasia and mucosal } \\
\text { adenocarcinoma in } \\
\text { Barrett's oesophagus. } \\
\text { Gut 2008; 57: 1200- } \\
1206 \text {. }\end{array}$ & & & & & & & \\
\hline 48 & $\begin{array}{l}\text { A randomized study of } \\
\text { chemotherapy, radiation } \\
\text { therapy, and surgery } \\
\text { versus surgery for } \\
\text { localized squamous cell } \\
\text { carcinoma of the } \\
\text { esophagus. Cancer } \\
\text { 1994; } 73 \text { : } 1779-1784 \text {. }\end{array}$ & France & 450 & 17.31 & ESCC & RCT & Chemoradiotherapy & Preoperative \\
\hline 49 & $\begin{array}{l}\text { Prospective study of } \\
\text { risk factors for } \\
\text { esophageal and gastric } \\
\text { cancers in the Linxian } \\
\text { General Population Trial } \\
\text { cohort in China. } \\
\text { International Journal of } \\
\text { Cancer 2005; 113: 456- } \\
463 \text {. }\end{array}$ & USA & 449 & 29.93 & ESCC & Cohort study & $\begin{array}{l}\text { Pathogenesis/Clinical } \\
\text { presentation }\end{array}$ & - \\
\hline 50 & $\begin{array}{l}\text { Endoscopic } \\
\text { submucosal dissection } \\
\text { of early esophageal } \\
\text { cancer. Clinical } \\
\text { gastroenterology and } \\
\text { hepatology : the official } \\
\text { clinical practice journal } \\
\text { of the American } \\
\text { Gastroenterological } \\
\text { Association 2005; } 3 \text { : } \\
\text { S67-70. }\end{array}$ & Japan & 446 & 29.73 & ESCC & $\begin{array}{l}\text { Prospective } \\
\text { study }\end{array}$ & Surgery & $\begin{array}{l}\text { Radical } \\
\text { Treatment }\end{array}$ \\
\hline 51 & $\begin{array}{l}\text { The incidence of } \\
\text { adenocarcinoma in } \\
\text { Barrett's esophagus: A } \\
\text { prospective study of } \\
170 \text { patients followed } \\
4.8 \text { years. American } \\
\text { Journal of } \\
\text { Gastroenterology 1997; } \\
\text { 92: } 212-215 \text {. }\end{array}$ & USA & 443 & 19.26 & $\mathrm{AE}$ & Cohort study & $\begin{array}{l}\text { Pathogenesis/Clinical } \\
\text { presentation }\end{array}$ & - \\
\hline 52 & $\begin{array}{l}\text { Intratumor } \\
\text { Heterogeneity } \\
\text { Characterized by } \\
\text { Textural Features on } \\
\text { Baseline F-18-FDG PET } \\
\text { Images Predicts } \\
\text { Response to } \\
\text { Concomitant } \\
\text { Radiochemotherapy in } \\
\text { Esophageal Cancer. } \\
\text { Journal of Nuclear } \\
\text { Medicine } 2011 ; 52: 369- \\
378 \text { - }\end{array}$ & France & 442 & 49.11 & Ade+ESCC & $\begin{array}{l}\text { Prospective } \\
\text { study }\end{array}$ & Imaging & - \\
\hline 53 & $\begin{array}{l}\text { Exome and whole- } \\
\text { genome sequencing of } \\
\text { esophageal } \\
\text { adenocarcinoma } \\
\text { identifies recurrent } \\
\text { driver events and } \\
\text { mutational complexity. } \\
\text { Nature Genetics 2013; } \\
\text { 45: } 478-\cup 437 \text {. }\end{array}$ & USA & 438 & 62.57 & $\mathrm{AE}$ & $\begin{array}{l}\text { Laboratory } \\
\text { investigation }\end{array}$ & $\begin{array}{l}\text { Pathogenesis/Clinical } \\
\text { presentation }\end{array}$ & - \\
\hline 54 & $\begin{array}{l}\text { Frequent mutation of } \\
\text { the p53 gene in human } \\
\text { esophageal cancer. } \\
\text { Proceedings of the } \\
\text { National Academy of } \\
\text { Sciences of the United } \\
\text { States of America 1990; } \\
\text { 87: } 9958-9961 \text {. }\end{array}$ & USA & 429 & 14.30 & ESCC & $\begin{array}{l}\text { Laboratory } \\
\text { investigation }\end{array}$ & $\begin{array}{l}\text { Pathogenesis/Clinical } \\
\text { presentation }\end{array}$ & - \\
\hline 55 & $\begin{array}{l}\text { Improvement in the } \\
\text { results of surgical } \\
\text { treatment of advanced }\end{array}$ & Japan & 427 & 21.35 & ESCC & $\begin{array}{l}\text { Retrospective } \\
\text { study }\end{array}$ & Surgery & $\begin{array}{l}\text { Radical } \\
\text { treatment }\end{array}$ \\
\hline
\end{tabular}

Page 13/25 


\begin{tabular}{|c|c|c|c|c|c|c|c|c|}
\hline & $\begin{array}{l}\text { squamous esophageal } \\
\text { carcinoma during } 15 \\
\text { consecutive years. } \\
\text { Annals of Surgery 2000; } \\
\text { 232: } 225-232 \text {. }\end{array}$ & & & & & & & \\
\hline 56 & $\begin{array}{l}\text { Results of a nationwide } \\
\text { study on the three-field } \\
\text { lymph node dissection } \\
\text { of esophageal cancer. } \\
\text { Oncology 1991; 48: } 411 \text { - } \\
420 \text {. }\end{array}$ & Japan & 417 & 14.38 & ESCC & $\begin{array}{l}\text { Retrospective } \\
\text { study }\end{array}$ & Surgery & $\begin{array}{l}\text { Radical } \\
\text { treatment }\end{array}$ \\
\hline 57 & $\begin{array}{l}\text { Increased populations } \\
\text { of regulatory T cells in } \\
\text { peripheral blood and } \\
\text { tumor-infiltrating } \\
\text { lymphocytes in patients } \\
\text { with gastric and } \\
\text { esophageal cancers. } \\
\text { Clinical Cancer } \\
\text { Research 2003; 9: 4404- } \\
4408 \text {. }\end{array}$ & Japan & 413 & 24.29 & ESCC & $\begin{array}{l}\text { Laboratory } \\
\text { investigation }\end{array}$ & $\begin{array}{l}\text { Pathogenesis/Clinical } \\
\text { presentation }\end{array}$ & - \\
\hline 58 & $\begin{array}{l}\text { Safety and Efficacy of } \\
\text { Pembrolizumab } \\
\text { Monotherapy in } \\
\text { Patients With Previously } \\
\text { Treated Advanced } \\
\text { Gastric and } \\
\text { Gastroesophageal } \\
\text { Junction Cancer Phase } \\
\text { 2 Clinical KEYNOTE-059 } \\
\text { Trial. Jama Oncology } \\
\text { 2018; } 4 \text {. }\end{array}$ & USA & 407 & 203.50 & $A E G+G$ & $\begin{array}{l}\text { Prospective } \\
\text { study }\end{array}$ & $\begin{array}{l}\text { New } \\
\text { agents_immunotherapy }\end{array}$ & Salvage \\
\hline 59 & $\begin{array}{l}\text { Obesity, alcohol, and } \\
\text { tobacco as risk factors } \\
\text { for cancers of the } \\
\text { esophagus and gastric } \\
\text { cardia: adenocarcinoma } \\
\text { versus squamous cell } \\
\text { carcinoma. Cancer } \\
\text { Epidemiology } \\
\text { Biomarkers \& } \\
\text { Prevention 1995; 4: 85- } \\
\text { 92. }\end{array}$ & USA & 405 & 16.20 & Ade+ESCC & $\begin{array}{l}\text { Case-control } \\
\text { study }\end{array}$ & $\begin{array}{l}\text { Pathogenesis/Clinical } \\
\text { presentation }\end{array}$ & - \\
\hline 60 & $\begin{array}{l}\text { Prospective randomized } \\
\text { trial comparing } \\
\text { mitomycin, cisplatin, } \\
\text { and protracted venous- } \\
\text { infusion fluorouracil } \\
\text { (PV15-FU) with } \\
\text { epirubicin, cisplatin, and } \\
\text { PV15-FU in advanced } \\
\text { esophagogastric cancer. } \\
\text { Journal of Clinical } \\
\text { Oncology 2002; 20: } \\
\text { 1996-2004. }\end{array}$ & UK & 398 & 22.11 & Ade+ESCC & RCT & Chemotherapy & Salvage \\
\hline 61 & $\begin{array}{l}\text { Utility of positron } \\
\text { emission tomography } \\
\text { for the staging of } \\
\text { patients with potentially } \\
\text { operable esophageal } \\
\text { carcinoma. Journal of } \\
\text { Clinical Oncology 2000; } \\
\text { 18: } 3202-3210 \text {. }\end{array}$ & Belgium & 392 & 19.60 & Ade+ESCC & $\begin{array}{l}\text { Prospective } \\
\text { study }\end{array}$ & Imaging & - \\
\hline 62 & $\begin{array}{l}\text { Curative endoscopic } \\
\text { resection of early } \\
\text { esophageal } \\
\text { adenocarcinomas } \\
\text { (Barrett's cancer). } \\
\text { Gastrointestinal } \\
\text { Endoscopy 2007; 65: 3- } \\
10 .\end{array}$ & Germany & 391 & 30.08 & $\mathrm{AE}$ & $\begin{array}{l}\text { Prospective } \\
\text { study }\end{array}$ & Surgery & $\begin{array}{l}\text { Radical } \\
\text { treatment }\end{array}$ \\
\hline 63 & $\begin{array}{l}\text { Histologic tumor type is } \\
\text { an independent } \\
\text { prognostic parameter in } \\
\text { esophageal cancer: } \\
\text { Lessons from more } \\
\text { than } 1,000 \text { consecutive } \\
\text { resections at a single }\end{array}$ & Germany & 391 & 20.58 & Ade+ESCC & $\begin{array}{l}\text { Retrospective } \\
\text { study }\end{array}$ & Surgery & $\begin{array}{l}\text { Radical } \\
\text { treatment }\end{array}$ \\
\hline
\end{tabular}

Page 14/25 
center in the Western

world. Annals of

Surgery 2001; 234: 360-

367.

\begin{tabular}{|c|c|c|c|c|c|c|c|c|}
\hline 64 & $\begin{array}{l}\text { The Number of Lymph } \\
\text { Nodes Removed } \\
\text { Predicts Survival in } \\
\text { Esophageal Cancer: An } \\
\text { International Study on } \\
\text { the Impact of Extent of } \\
\text { Surgical Resection. } \\
\text { Annals of Surgery 2008; } \\
\text { 248: 549-554. }\end{array}$ & USA & 381 & 31.75 & Ade+ESCC & $\begin{array}{l}\text { Retrospective } \\
\text { study }\end{array}$ & Surgery & $\begin{array}{l}\text { Radical } \\
\text { treatment }\end{array}$ \\
\hline 65 & $\begin{array}{l}\text { miR-145, miR-133a and } \\
\text { miR-133b: tumor- } \\
\text { suppressive miRNAs } \\
\text { target FSCN1 in } \\
\text { esophageal squamous } \\
\text { cell carcinoma. } \\
\text { International Journal of } \\
\text { Cancer 2010; 127: 2804- } \\
\text { 2814. }\end{array}$ & Japan & 381 & 38.10 & ESCC & $\begin{array}{l}\text { Laboratory } \\
\text { investigation }\end{array}$ & $\begin{array}{l}\text { Pathogenesis/Clinical } \\
\text { presentation }\end{array}$ & - \\
\hline 66 & $\begin{array}{l}\text { Specialized intestinal } \\
\text { metaplasia, dysplasia, } \\
\text { and cancer of the } \\
\text { esophagus and } \\
\text { esophagogastric } \\
\text { junction: Prevalence } \\
\text { and clinical data. } \\
\text { Gastroenterology 1999; } \\
\text { 116: } 277-285 \text {. }\end{array}$ & USA & 379 & 18.05 & $\mathrm{AE}$ & $\begin{array}{l}\text { Retrospective } \\
\text { study }\end{array}$ & $\begin{array}{l}\text { Pathogenesis/Clinical } \\
\text { presentation }\end{array}$ & - \\
\hline 67 & $\begin{array}{l}\text { Prognostic significance } \\
\text { of activated CD 8(+) T } \\
\text { cell infiltrations within } \\
\text { esophageal } \\
\text { carcinomas. Cancer } \\
\text { Research 2001; 61: } \\
\text { 3932-3936. }\end{array}$ & Germany & 378 & 19.89 & Ade+ESCC & $\begin{array}{l}\text { Laboratory } \\
\text { investigation }\end{array}$ & $\begin{array}{l}\text { Pathogenesis/Clinical } \\
\text { presentation }\end{array}$ & - \\
\hline 68 & $\begin{array}{l}\text { Time course of tumor } \\
\text { metabolic activity } \\
\text { during } \\
\text { chemoradiotherapy of } \\
\text { esophageal squamous } \\
\text { cell carcinoma and } \\
\text { response to treatment. } \\
\text { Journal of Clinical } \\
\text { Oncology 2004; } 22 \text { : } 900- \\
\text { 908. }\end{array}$ & Germany & 371 & 23.19 & ESCC & $\begin{array}{l}\text { Prospective } \\
\text { study }\end{array}$ & Imaging & - \\
\hline 69 & $\begin{array}{l}\text { A shared susceptibility } \\
\text { locus in PLCE1 at 10q23 } \\
\text { for gastric } \\
\text { adenocarcinoma and } \\
\text { esophageal squamous } \\
\text { cell carcinoma. Nature } \\
\text { Genetics 2010; 42: 764- } \\
\text { U751. }\end{array}$ & USA & 369 & 36.90 & Ade+ESCC & $\begin{array}{l}\text { Laboratory } \\
\text { investigation }\end{array}$ & $\begin{array}{l}\text { Pathogenesis/Clinical } \\
\text { presentation }\end{array}$ & - \\
\hline 70 & $\begin{array}{l}\text { Use of aspirin and other } \\
\text { nonsteroidal anti- } \\
\text { inflammatory drugs and } \\
\text { risk of esophageal and } \\
\text { gastric cancer. Cancer } \\
\text { Epidemiology } \\
\text { Biomarkers \& } \\
\text { Prevention 1998; 7: 97- } \\
\text { 102. }\end{array}$ & USA & 367 & 16.68 & Ade+ESCC & $\begin{array}{l}\text { Case-control } \\
\text { study }\end{array}$ & $\begin{array}{l}\text { Pathogenesis/Clinical } \\
\text { presentation }\end{array}$ & - \\
\hline 71 & $\begin{array}{l}\text { Expression of survivin in } \\
\text { esophageal cancer: } \\
\text { Correlation with the } \\
\text { prognosis and response } \\
\text { to chemotherapy. } \\
\text { International Journal of } \\
\text { Cancer 2001; } 95: 92-95 \text {. }\end{array}$ & Japan & 362 & 19.05 & ESCC & $\begin{array}{l}\text { Laboratory } \\
\text { investigation }\end{array}$ & $\begin{array}{l}\text { Pathogenesis/Clinical } \\
\text { presentation }\end{array}$ & - \\
\hline 72 & $\begin{array}{l}\text { Complete response to } \\
\text { neoadjuvant } \\
\text { chemoradiotherapy in } \\
\text { esophageal carcinoma } \\
\text { is associated with }\end{array}$ & USA & 356 & 23.73 & Ade+ESCC & $\begin{array}{l}\text { Retrospective } \\
\text { study }\end{array}$ & Chemoradiotherapy & Preoperative \\
\hline
\end{tabular}

Page 15/25 
significantly improved

survival. Journal of

Clinical Oncology 2005;

23: 4330-4337.

\begin{tabular}{|c|c|c|c|c|c|c|c|c|}
\hline 73 & $\begin{array}{l}\text { Long-term results of } \\
\text { RTOG trial 8911 (USA } \\
\text { intergroup 113): A } \\
\text { random assignment } \\
\text { trial comparison of } \\
\text { chemotherapy followed } \\
\text { by surgery compared } \\
\text { with surgery alone for } \\
\text { esophageal cancer. } \\
\text { Journal of Clinical } \\
\text { Oncology 2007; } 25 \text { : } \\
3719-3725 \text {. }\end{array}$ & USA & 349 & 26.85 & Ade+ESCC & $\mathrm{RCT}$ & Chemotherapy & Preoperative \\
\hline 74 & $\begin{array}{l}\text { Metabolic imaging } \\
\text { predicts response, } \\
\text { survival, and recurrence } \\
\text { in adenocarcinomas of } \\
\text { the esophagogastric } \\
\text { junction. Journal of } \\
\text { Clinical Oncology 2006; } \\
\text { 24: } 4692-4698 \text {. }\end{array}$ & Germany & 347 & 24.79 & $\mathrm{AE}$ & $\begin{array}{l}\text { Prospective } \\
\text { study }\end{array}$ & Imaging & - \\
\hline 75 & $\begin{array}{l}\text { Multivariate prognostic } \\
\text { factor analysis in locally } \\
\text { advanced and } \\
\text { metastatic esophago- } \\
\text { gastric cancer-pooled } \\
\text { analysis from three } \\
\text { multicenter, randomized, } \\
\text { controlled trials using } \\
\text { individual patient data. } \\
\text { Journal of Clinical } \\
\text { Oncology 2004; 22: } \\
\text { 2395-2403. }\end{array}$ & UK & 347 & 21.69 & Ade+ESCC & $\begin{array}{l}\text { Retrospective } \\
\text { study }\end{array}$ & Chemotherapy & Salvage \\
\hline 76 & $\begin{array}{l}\text { Multicenter phase II } \\
\text { study of irinotecan, } \\
\text { cisplatin, and } \\
\text { bevacizumab in } \\
\text { patients with metastatic } \\
\text { gastric or } \\
\text { gastroesophageal } \\
\text { junction } \\
\text { adenocarcinorna. } \\
\text { Journal of Clinical } \\
\text { Oncology 2006; 24: } \\
5201-5206 \text {. }\end{array}$ & USA & 346 & 24.71 & $A E G+G$ & $\begin{array}{l}\text { Prospective } \\
\text { study }\end{array}$ & Chemotherapy & Salvage \\
\hline 77 & $\begin{array}{l}\text { Adenocarcinoma of the } \\
\text { esophagogastric } \\
\text { junction and Barrett's } \\
\text { esophagus. } \\
\text { Gastroenterology 1995; } \\
\text { 109: 1541-1546. }\end{array}$ & USA & 345 & 13.80 & $\mathrm{AE}$ & $\begin{array}{l}\text { Case-control } \\
\text { study }\end{array}$ & $\begin{array}{l}\text { Pathogenesis/Clinical } \\
\text { presentation }\end{array}$ & - \\
\hline 78 & $\begin{array}{l}\text { Early esophageal cancer } \\
\text { - Pattern of lymphatic } \\
\text { spread and prognostic } \\
\text { factors for long-term } \\
\text { survival after surgical } \\
\text { resection. Annals of } \\
\text { Surgery 2005; } 242: 566 \text { - } \\
575 \text {. }\end{array}$ & Germany & 339 & 22.60 & Ade+ESCC & $\begin{array}{l}\text { Retrospective } \\
\text { study }\end{array}$ & Surgery & $\begin{array}{l}\text { Radical } \\
\text { treatment }\end{array}$ \\
\hline 79 & $\begin{array}{l}\text { Prognostic factors for } \\
\text { the survival of patients } \\
\text { with esophageal } \\
\text { carcinoma in the US - } \\
\text { The importance of } \\
\text { tumor length and lymph } \\
\text { node status. Cancer } \\
\text { 2002; } 95: 1434-1443 \text {. }\end{array}$ & USA & 339 & 18.83 & Ade+ESCC & $\begin{array}{l}\text { Retrospective } \\
\text { study }\end{array}$ & Surgery & $\begin{array}{l}\text { Radical } \\
\text { treatment }\end{array}$ \\
\hline 80 & $\begin{array}{l}\text { MicroRNA expression } \\
\text { profiles of esophageal } \\
\text { cancer. Journal of } \\
\text { Thoracic and } \\
\text { Cardiovascular Surgery } \\
\text { 2008; } 135 \text { : } 255-260 \text {. }\end{array}$ & USA & 339 & 28.25 & Ade+ESCC & $\begin{array}{l}\text { Laboratory } \\
\text { investigation }\end{array}$ & $\begin{array}{l}\text { Pathogenesis/Clinical } \\
\text { presentation }\end{array}$ & - \\
\hline 81 & Esophageal cancer: & USA & 337 & 16.85 & Ade+ESCC & Retrospective & Pathogenesis/Clinical & - \\
\hline
\end{tabular}

Page 16/25 


\begin{tabular}{|c|c|c|c|c|c|c|c|c|}
\hline & $\begin{array}{l}\text { Results of an American } \\
\text { College of Surgeons } \\
\text { patient care evaluation } \\
\text { study. Journal of the } \\
\text { American College of } \\
\text { Surgeons 2000; } 190 \text { : } \\
562-572 \text {. }\end{array}$ & & & & & study & presentation & \\
\hline 82 & $\begin{array}{l}\text { Three-field lymph node } \\
\text { dissection for } \\
\text { squamous cell and } \\
\text { adenocarcinoma of the } \\
\text { esophagus. Annals of } \\
\text { Surgery 2002; 236: 177- } \\
\text { 183. }\end{array}$ & USA & 336 & 18.67 & Ade+ESCC & $\begin{array}{l}\text { Prospective } \\
\text { study }\end{array}$ & $\begin{array}{l}\text { Pathogenesis/Clinical } \\
\text { presentation }\end{array}$ & - \\
\hline 83 & $\begin{array}{l}\text { Endoscopic biopsy can } \\
\text { detect high-grade } \\
\text { dysplasia or early } \\
\text { adenocarcinoma in } \\
\text { Barrett's esophagus } \\
\text { without grossly } \\
\text { recognizable neoplastic } \\
\text { lesions. } \\
\text { Gastroenterology 1988; } \\
\text { 94: 81-90. }\end{array}$ & USA & 336 & 10.50 & $\mathrm{AE}$ & $\begin{array}{l}\text { Prospective } \\
\text { study }\end{array}$ & $\begin{array}{l}\text { Pathogenesis/Clinical } \\
\text { presentation }\end{array}$ & - \\
\hline 84 & $\begin{array}{l}\text { Nutrition intervention } \\
\text { trials in Linxian, China: } \\
\text { multiple } \\
\text { vitamin/mineral } \\
\text { supplementation, } \\
\text { cancer incidence, and } \\
\text { disease-specific } \\
\text { mortality among adults } \\
\text { with esophageal } \\
\text { dysplasia. Journal of } \\
\text { the National Cancer } \\
\text { Institute 1993; } 85: 1492- \\
1498 \text {. }\end{array}$ & China & 335 & 12.41 & ESCC & $\mathrm{RCT}$ & $\begin{array}{l}\text { Pathogenesis/Clinical } \\
\text { presentation }\end{array}$ & $\begin{array}{l}\text { Nutrition } \\
\text { intervention }\end{array}$ \\
\hline 85 & $\begin{array}{l}\text { Nutrient intake and risk } \\
\text { of subtypes of } \\
\text { esophageal and gastric } \\
\text { cancer. Cancer } \\
\text { Epidemiology } \\
\text { Biomarkers \& } \\
\text { Prevention 2001; } 10 \text { : } \\
\text { 1055-1062. }\end{array}$ & USA & 333 & 17.53 & Ade+ESCC & $\begin{array}{l}\text { Retrospective } \\
\text { study }\end{array}$ & $\begin{array}{l}\text { Pathogenesis/Clinical } \\
\text { presentation }\end{array}$ & - \\
\hline 86 & $\begin{array}{l}\text { Early Detection of } \\
\text { Superficial Squamous } \\
\text { Cell Carcinoma in the } \\
\text { Head and Neck Region } \\
\text { and Esophagus by } \\
\text { Narrow Band Imaging: A } \\
\text { Multicenter Randomized } \\
\text { Controlled Trial. Journal } \\
\text { of Clinical Oncology } \\
2010 ; 28: 1566-1572 \text {. }\end{array}$ & Japan & 332 & 33.20 & ESCC & RCT & Imaging & - \\
\hline 87 & $\begin{array}{l}\text { Principles of surgical } \\
\text { treatment for carcinoma } \\
\text { of the esophagus: } \\
\text { analysis of lymph node } \\
\text { involvement. Annals of } \\
\text { surgery } 1981 ; 194: 438- \\
446 \text {. }\end{array}$ & Japan & 331 & 8.49 & ESCC & $\begin{array}{l}\text { Retrospective } \\
\text { study }\end{array}$ & $\begin{array}{l}\text { Pathogenesis/Clinical } \\
\text { presentation }\end{array}$ & \\
\hline 88 & $\begin{array}{l}\text { Cancer of the } \\
\text { Esophagus and } \\
\text { Esophagogastric } \\
\text { Junction Data-Driven } \\
\text { Staging for the Seventh } \\
\text { Edition of the American } \\
\text { Joint Committee on } \\
\text { Cancer/International } \\
\text { Union Against Cancer } \\
\text { Cancer Staging } \\
\text { Manuals. Cancer 2010; } \\
\text { 116: 3763-3773. }\end{array}$ & USA & 331 & 33.10 & Ade+ESCC & $\begin{array}{l}\text { Retrospective } \\
\text { study }\end{array}$ & $\begin{array}{l}\text { Pathogenesis/Clinical } \\
\text { presentation }\end{array}$ & - \\
\hline 89 & $\begin{array}{l}\text { Genome-wide } \\
\text { association study of } \\
\text { esophageal squamous } \\
\text { cell carcinoma in }\end{array}$ & China & 331 & 33.10 & ESCC & $\begin{array}{l}\text { Laboratory } \\
\text { investigation }\end{array}$ & $\begin{array}{l}\text { Pathogenesis/Clinical } \\
\text { presentation }\end{array}$ & - \\
\hline
\end{tabular}

Page $17 / 25$ 
Chinese subjects

identifies susceptibility

loci at PLCE1 and

C20orf54. Nature

Genetics 2010; 42: 759-

U746.

\begin{tabular}{|c|c|c|c|c|c|c|c|c|}
\hline 90 & $\begin{array}{l}\text { MicroRNA Expression in } \\
\text { Squamous Cell } \\
\text { Carcinoma and } \\
\text { Adenocarcinoma of the } \\
\text { Esophagus: } \\
\text { Associations with } \\
\text { Survival. Clinical Cancer } \\
\text { Research 2009; 15: } \\
\text { 6192-6200. }\end{array}$ & USA & 331 & 30.09 & Ade+ESCC & $\begin{array}{l}\text { Laboratory } \\
\text { investigation }\end{array}$ & $\begin{array}{l}\text { Pathogenesis/Clinical } \\
\text { presentation }\end{array}$ & - \\
\hline 91 & $\begin{array}{l}\text { Definitive } \\
\text { chemoradiotherapy for } \\
\text { T4 and/or M1 lymph } \\
\text { node squamous cell } \\
\text { carcinoma of the } \\
\text { esophagus. Journal of } \\
\text { Clinical Oncology } 1999 ; \\
\text { 17: } 2915-2921 \text {. }\end{array}$ & Japan & 330 & 15.71 & ESCC & $\begin{array}{l}\text { Retrospective } \\
\text { study }\end{array}$ & Chemoradiotherapy & $\begin{array}{l}\text { Radical } \\
\text { Treatment }\end{array}$ \\
\hline 92 & $\begin{array}{l}\text { MET Amplification } \\
\text { Identifies a Small and } \\
\text { Aggressive Subgroup of } \\
\text { Esophagogastric } \\
\text { Adenocarcinoma With } \\
\text { Evidence of } \\
\text { Responsiveness to } \\
\text { Crizotinib. Journal of } \\
\text { Clinical Oncology 2011; } \\
\text { 29: } 4803-4810 \text {. }\end{array}$ & USA & 328 & 36.44 & $\mathrm{AE}$ & $\begin{array}{l}\text { Laboratory } \\
\text { investigation }\end{array}$ & $\begin{array}{l}\text { Pathogenesis/Clinical } \\
\text { presentation }\end{array}$ & - \\
\hline 93 & $\begin{array}{l}\text { Only pathologic } \\
\text { complete response to } \\
\text { neoadjuvant } \\
\text { chemotherapy improves } \\
\text { significantly the long } \\
\text { term survival of patients } \\
\text { with resectable } \\
\text { esophageal squamous } \\
\text { cell carcinoma - Final } \\
\text { report of a randomized, } \\
\text { controlled trial of } \\
\text { preoperative } \\
\text { chemotherapy versus } \\
\text { surgery alone. Cancer } \\
\text { 2001; } 91: 2165-2174 \text {. }\end{array}$ & Italy & 326 & 17.16 & ESCC & $\mathrm{RCT}$ & Chemotherapy & Preoperative \\
\hline 94 & $\begin{array}{l}\text { Docetaxel versus active } \\
\text { symptom control for } \\
\text { refractory } \\
\text { oesophagogastric } \\
\text { adenocarcinoma } \\
\text { (COUGAR-02): an open- } \\
\text { label, phase } 3 \\
\text { randomised controlled } \\
\text { trial. Lancet Oncology } \\
\text { 2014; } 15 \text { : 78-86. }\end{array}$ & UK & 324 & 54.00 & $\mathrm{AE}$ & $\mathrm{RCT}$ & Chemotherapy & Salvage \\
\hline 95 & $\begin{array}{l}\text { Mortality and morbidity } \\
\text { rates, postoperative } \\
\text { course, quality of life, } \\
\text { and prognosis after } \\
\text { extended radical } \\
\text { lymphadenectomy for } \\
\text { esophageal cancer. } \\
\text { Comparison of three- } \\
\text { field lymphadenectomy } \\
\text { with two-field } \\
\text { lymphadenectomy. } \\
\text { Annals of Surgery 1995; } \\
\text { 222: 654-662. }\end{array}$ & Japan & 323 & 12.92 & ESCC & $\begin{array}{l}\text { Prospective } \\
\text { study }\end{array}$ & Surgery & $\begin{array}{l}\text { Radical } \\
\text { treatment }\end{array}$ \\
\hline 96 & $\begin{array}{l}\text { Time trends incidence } \\
\text { of both major histologic } \\
\text { types of esophageal } \\
\text { carcinomas in selected } \\
\text { countries, 1973-1995. } \\
\text { International Journal of } \\
\text { Cancer 2002; 99: 860- } \\
868 \text {. }\end{array}$ & France & 323 & 17.94 & Ade+ESCC & $\begin{array}{l}\text { Cross- } \\
\text { sectional } \\
\text { study }\end{array}$ & $\begin{array}{l}\text { Pathogenesis/Clinical } \\
\text { presentation }\end{array}$ & - \\
\hline
\end{tabular}

Page 18/25 


\begin{tabular}{|c|c|c|c|c|c|c|c|c|}
\hline 97 & $\begin{array}{l}\text { Preoperative } \\
\text { chemoradiation } \\
\text { followed by transhiatal } \\
\text { esophagectomy for } \\
\text { carcinoma of the } \\
\text { esophagus: final report. } \\
\text { Journal of Clinical } \\
\text { Oncology 1993; } 11 \text { : } \\
\text { 1118-1123. }\end{array}$ & USA & 321 & 11.89 & Ade+ESCC & $\begin{array}{l}\text { Prospective } \\
\text { study }\end{array}$ & Chemoradiotherapy & Preoperative \\
\hline 98 & $\begin{array}{l}\text { Randomized clinical } \\
\text { trial of preoperative and } \\
\text { postoperative adjuvant } \\
\text { chemotherapy with } \\
\text { cisplatin, vindesine, and } \\
\text { bleomycin for } \\
\text { carcinoma of the } \\
\text { esophagus. The Journal } \\
\text { of thoracic and } \\
\text { cardiovascular surgery } \\
\text { 1988; } 96: 242-248 \text {. }\end{array}$ & USA & 319 & 9.97 & Ade+ESCC & $\mathrm{RCT}$ & Chemotherapy & Perioperative \\
\hline 99 & $\begin{array}{l}\text { Three-field } \\
\text { lymphadenectomy for } \\
\text { carcinoma of the } \\
\text { esophagus and } \\
\text { gastroesophageal } \\
\text { junction in } 174 \text { R-0 } \\
\text { resections: Impact on } \\
\text { staging, disease-free } \\
\text { survival, and outcome- } \\
\text { A plea for adaptation of } \\
\text { TNM classification in } \\
\text { upper-half esophageal } \\
\text { carcinoma. Annals of } \\
\text { Surgery 2004; } 240: 962- \\
974 \text {. }\end{array}$ & Belgium & 315 & 19.69 & Ade+ESCC & $\begin{array}{l}\text { Retrospective } \\
\text { study }\end{array}$ & $\begin{array}{l}\text { Pathogenesis/Clinical } \\
\text { presentation }\end{array}$ & - \\
\hline 100 & $\begin{array}{l}\text { Minimally invasive } \\
\text { esophagectomy: } \\
\text { Thoracoscopic } \\
\text { mobilization of the } \\
\text { esophagus and } \\
\text { mediastinal } \\
\text { lymphadenectomy in } \\
\text { prone position - } \\
\text { Experience of } 130 \\
\text { patients. Journal of the } \\
\text { American College of } \\
\text { Surgeons 2006; 203: 7- } \\
\text { 16. }\end{array}$ & India & 314 & 22.43 & ESCC & $\begin{array}{l}\text { Prospective } \\
\text { study }\end{array}$ & Surgery & $\begin{array}{l}\text { Radical } \\
\text { Treatment }\end{array}$ \\
\hline
\end{tabular}

$\mathrm{AE}$, adenocarcinoma; $\mathrm{AEG}$, adenocarcinoma of the esophagogastric junction; $\mathrm{G}$, gastric adenocarcinoma; ESCC, esophageal squamous cell carcinoma; ADE, adenocarcinoma of the esophagus; RCT, randomized controlled trial; USA, United States of America; UK, United Kingdom

Table 2. Journals in which the 100 most-cited articles were published. 


\begin{tabular}{|c|c|c|c|c|}
\hline $\begin{array}{l}\text { Abbreviated journal name (International } \\
\text { Organization for Standardization) }\end{array}$ & Journal category & $\begin{array}{l}\text { Number of articles } \\
\text { published }\end{array}$ & $\begin{array}{l}\text { Average number of } \\
\text { citations per paper }\end{array}$ & $\begin{array}{l}\text { Impact } \\
\text { factor }\end{array}$ \\
\hline J Clin Oncol & Oncology & 26 & 547 & 32.956 \\
\hline Ann Surg & Surgery & 10 & 385 & 10.130 \\
\hline N Engl J Med & General & 9 & 1819 & 74.699 \\
\hline Lancet Oncol & Oncology & 6 & 655 & 33.752 \\
\hline Lancet & General & 5 & 813 & 60.392 \\
\hline Cancer & Oncology & 5 & 539 & 5.742 \\
\hline Nature Genet. & Genetics\&Heredity & 4 & 446 & 27.603 \\
\hline Gastroenterology & Gastroenterology\&Hepatology & 4 & 385 & 17.373 \\
\hline Int. J. Cancer & Oncology & 4 & 379 & 5.145 \\
\hline Clin Cancer Res & Oncology & 3 & 428.3 & 10.107 \\
\hline $\begin{array}{l}\text { Cancer epidemiol. } \\
\text { Biomarkers Prev. }\end{array}$ & $\begin{array}{l}\text { Public, Environmental \& } \\
\text { Occupation Health }\end{array}$ & 3 & 368.3 & 4.344 \\
\hline Cancer Res & Oncology & 2 & 418.5 & 9.727 \\
\hline JNCl-J Natl Cancer Inst & Oncology & 2 & 634.5 & 11.577 \\
\hline JAMA-J Am Med Assoc & General & 2 & 1662 & 45.540 \\
\hline J. Am. Coll. Surg. & Surgery & 2 & 325.5 & 4.590 \\
\hline J.Thorac. Cardiovasc. Surg. & Surgery & 2 & 329 & 4.451 \\
\hline Gut & Gastroenterology \& Hepatology & 1 & 455 & 19.819 \\
\hline Ann. Surg. Oncol. & Oncology & 1 & 585 & 4.061 \\
\hline Am. J. Gastroenterol. & Gastroenterology \& Hepatology & 1 & 443 & 10.171 \\
\hline \multirow[t]{2}{*}{ Appl. Enviro. Microbiol. } & Microbiology & 1 & 470 & 4.016 \\
\hline & $\begin{array}{l}\text { Biotechnology \& Applied } \\
\text { microbiology }\end{array}$ & & & \\
\hline Gastrointest. Endosc. & Gastroenterology \& Hepatology & 1 & 391 & 6.890 \\
\hline J. Nucl. med. & $\begin{array}{l}\text { Radiology, nuclear medicine \& } \\
\text { medical imaging }\end{array}$ & 1 & 442 & 7.887 \\
\hline PNAS & General & 1 & 429 & 9.580 \\
\hline World J. Surg. & Surgery & 1 & 465 & 2.234 \\
\hline Clin. Gastroenterol. Hepatol. & Gastroenterology \& Hepatology & 1 & 446 & 8.549 \\
\hline JAMA Oncol. & Oncology & 1 & 407 & 24.799 \\
\hline Oncology & Oncology & 1 & 417 & 2.642 \\
\hline
\end{tabular}

\section{References}

[1] Bray F, Ferlay J, Soerjomataram I, et al. Global cancer statistics 2018: GLOBOCAN estimates of incidence and mortality worldwide for 36 cancers in 185 countries[J]. CA Cancer J Clin. 2018, 68(6): 394-424.

[2] Chen W, Zheng R, Baade P D, et al. Cancer statistics in China, 2015[J]. CA: A Cancer Journal for Clinicians. 2016, 66(2): 115-132.

[3] Moed H F. New developments in the use of citation analysis in research evaluation[J]. Arch Immunol Ther Exp (Warsz). 2009, 57(1): 13-18.

[4] Garfield E. 100 citation classics from the Journal of the American Medical Association[J]. JAMA. 1987, 257(1): 52-59.

[5] Malhotra K, Saeed O, Goyal N, et al. Top-100 Highest-Cited Original Articles in Ischemic Stroke: A Bibliometric Analysis[J]. World Neurosurg. 2018, 111: e649-e660.

[6] Baltussen A, Kindler C H. Citation classics in critical care medicine[J]. Intensive Care Med. 2004, 30(5): $902-910$. 
[7] Guo X, Gao L, Wang Z, et al. Top 100 Most-Cited Articles on Pituitary Adenoma: A Bibliometric Analysis[J]. World Neurosurg. 2018, 116: e1153e1167.

[8] Kim H J, Yoon D Y, Kim E S, et al. The 100 most-cited articles in neuroimaging: A bibliometric analysis[J]. Neuroimage. 2016, 139: 149-156.

[9] Hachem L D, Mansouri A, Juraschka K, et al. Citation classics in neuro-oncology: assessment of historical trends and scientific progress[J]. Neuro Oncol. 2017, 19(9): 1158-1172.

[10] Malhotra K, Saeed O, Goyal N, et al. Top-100 Highest-Cited Original Articles in Ischemic Stroke: A Bibliometric Analysis[J]. World Neurosurg. 2018, 111: e649-e660.

[11] Brandt J S, Hadaya O, Schuster M, et al. A Bibliometric Analysis of Top-Cited Journal Articles in Obstetrics and Gynecology[J]. JAMA Netw Open. 2019, 2(12): e1918007.

[12] Gupta A, Kennedy B, Meriwether K V, et al. Citation classics: the 100 most cited articles in Urogynecology[J]. Int Urogynecol J. 2020, 31 (2): 249-266.

[13] Cunningham D, Allum W H, Stenning S P, et al. Perioperative chemotherapy versus surgery alone for resectable gastroesophageal cancer[J]. N Engl J Med. 2006, 355(1): 11-20.

[14] Macdonald J S, Smalley S R, Benedetti J, et al. Chemoradiotherapy after surgery compared with surgery alone for adenocarcinoma of the stomach or gastroesophageal junction[J]. N Engl J Med. 2001, 345(10): 725-730.

[15] van Hagen P, Hulshof M C, van Lanschot J J, et al. Preoperative chemoradiotherapy for esophageal or junctional cancer[J]. N Engl J Med. 2012, 366(22): 2074-2084.

[16] Hennessey K, Afshar K, Macneily A E. The top 100 cited articles in urology[J]. Can Urol Assoc J. 2009, 3(4): $293-302$.

[17] Galandi D, Schwarzer G, Antes G. The demise of the randomised controlled trial: bibliometric study of the German-language health care literature, 1948 to 2004[J]. BMC Med Res Methodol. 2006, 6: 30.

[18] Moher D, Pham B, Lawson M L, et al. The inclusion of reports of randomised trials published in languages other than English in systematic reviews[J]. Health Technol Assess. 2003, 7(41): 1-90.

[19] Al-Sarraf M, Martz K, Herskovic A, et al. Progress report of combined chemoradiotherapy versus radiotherapy alone in patients with esophageal cancer: an intergroup study[J]. J Clin Oncol. 1997, 15(1): 277-284.

[20] Cooper J S, Guo M D, Herskovic A, et al. Chemoradiotherapy of locally advanced esophageal cancer: long-term follow-up of a prospective randomized trial (RTOG 85-01). Radiation Therapy Oncology Group[J]. JAMA. 1999, 281(17): 1623-1627.

[21] Minsky B D, Pajak T F, Ginsberg R J, et al. INT 0123 (Radiation Therapy Oncology Group 94-05) phase III trial of combined-modality therapy for esophageal cancer: high-dose versus standard-dose radiation therapy[J]. J Clin Oncol. 2002, 20(5): 1167-1174.

[22] Isono K, Sato H, Nakayama K. Results of a nationwide study on the three-field lymph node dissection of esophageal cancer[J]. Oncology. 1991, 48(5): 411-420.

[23] Fujita H, Kakegawa T, Yamana H, et al. Mortality and morbidity rates, postoperative course, quality of life, and prognosis after extended radical lymphadenectomy for esophageal cancer. Comparison of three-field lymphadenectomy with two-field lymphadenectomy[J]. Ann Surg. 1995, 222(5): 654-662.

[24] Kato H, Watanabe H, Tachimori Y, et al. Evaluation of neck lymph node dissection for thoracic esophageal carcinoma[J]. Ann Thorac Surg. 1991, 51(6): 931-935.

[25] Nishihira T, Hirayama K, Mori S. A Prospective Randomized Trial of Extended Cervical and Superior Mediastinal Lymphadenectomy for Carcinoma of the Thoracic Esophagus[J]. Am J Surg. 1998, 175(1): 47-51.

[26] Tachibana M, Kinugasa S, Yoshimura H, et al. Clinical outcomes of extended esophagectomy with three-field lymph node dissection for esophageal squamous cell carcinoma[J]. The American Journal of Surgery. 2005, 189(1): 98-109.

[27] Lu J, Tao H, Song D, et al. Recurrence risk model for esophageal cancer after radical surgery[J]. Chin J Cancer Res. 2013, 25(5): 549-555.

[28] Miyata H, Yamasaki M, Kurokawa Y, et al. Survival Factors in Patients with Recurrence After Curative Resection of Esophageal Squamous Cell Carcinomas[J]. Ann Surg Oncol. 2011, 18(12): 3353-3361.

[29] Oppedijk V, van der Gaast A, van Lanschot J J B, et al. Patterns of Recurrence After Surgery Alone Versus Preoperative Chemoradiotherapy and Surgery in the CROSS Trials[J]. J Clin Oncol. 2014, 32(5): 385-391.

Page 21/25 
[30] Hsu P K, Wang B Y, Huang C S, et al. Prognostic factors for post-recurrence survival in esophageal squamous cell carcinoma patients with recurrence after resection[J]. J Gastrointest Surg. 2011, 15(4): 558-565.

[31] Pennathur A, Gibson M K, Jobe B A, et al. Oesophageal carcinoma[J]. Lancet. 2013, 381(9864): 400-412.

[32] Forastiere A A, Orringer M B, Perez-Tamayo C, et al. Preoperative chemoradiation followed by transhiatal esophagectomy for carcinoma of the esophagus: final report[J]. J Clin Oncol. 1993, 11(6): 1118-1123.

[33] Le Prise E, Etienne P L, Meunier B, et al. A randomized study of chemotherapy, radiation therapy, and surgery versus surgery for localized squamous cell carcinoma of the esophagus[J]. Cancer. 1994, 73(7): 1779-1784.

[34] Walsh T N, Noonan N, Hollywood D, et al. A Comparison of Multimodal Therapy and Surgery for Esophageal Adenocarcinoma - NEJM[J]. New England Journal of Medicine. 1996.

[35] Bosset J F, Gignoux M, Triboulet J P, et al. Chemoradiotherapy followed by surgery compared with surgery alone in squamous-cell cancer of the esophagus[J]. N Engl J Med. 1997, 337(3): 161-167.

[36] Urba S G, Orringer M B, Turrisi A, et al. Randomized trial of preoperative chemoradiation versus surgery alone in patients with locoregional esophageal carcinoma[J]. J Clin Oncol. 2001, 19(2): 305-313.

[37] Burmeister B H, Smithers B M, Gebski V, et al. Surgery alone versus chemoradiotherapy followed by surgery for resectable cancer of the oesophagus: a randomised controlled phase III trial[J]. Lancet Oncol. 2005, 6(9): 659-668.

[38] Shapiro J, van Lanschot J, Hulshof M, et al. Neoadjuvant chemoradiotherapy plus surgery versus surgery alone for oesophageal or junctional cancer (CROSS): long-term results of a randomised controlled trial[J]. Lancet Oncol. 2015, 16(9): 1090-1098.

[39] Roth J A, Pass H I, Flanagan M M, et al. Randomized clinical trial of preoperative and postoperative adjuvant chemotherapy with cisplatin, vindesine, and bleomycin for carcinoma of the esophagus[J]. J Thorac Cardiovasc Surg. 1988, 96(2): $242-248$.

[40] Ychou M, Boige V, Pignon J, et al. Perioperative Chemotherapy Compared With Surgery Alone for Resectable Gastroesophageal Adenocarcinoma: An FNCLCC and FFCD Multicenter Phase III Trial[J]. Journal of clinical oncology. 2011, 29(13): 1715-1721.

[41] Ando N, lizuka T, Ide H, et al. Surgery Plus Chemotherapy Compared With Surgery Alone for Localized Squamous Cell Carcinoma of the Thoracic Esophagus: A Japan Clinical Oncology Group Study-JCOG9204[J]. J Clin Oncol. 2003, 21(24): 4592-4596.

[42] Webb A, Cunningham D, Scarffe J H, et al. Randomized trial comparing epirubicin, cisplatin, and fluorouracil versus fluorouracil, doxorubicin, and methotrexate in advanced esophagogastric cancer[J]. J Clin Oncol. 1997, 15(1): 261-267.

[43] Ross P, Nicolson M, Cunningham D, et al. Prospective randomized trial comparing mitomycin, cisplatin, and protracted venous-infusion fluorouracil (PVI 5-FU) With epirubicin, cisplatin, and PVI 5-FU in advanced esophagogastric cancer[J]. J Clin Oncol. 2002, 20(8): $1996-2004$.

[44] Cunningham D, Starling N, Rao S, et al. Capecitabine and oxaliplatin for advanced esophagogastric cancer[J]. N Engl J Med. 2008, 358(1): 36-46.

[45] Bang Y J, Van Cutsem E, Feyereislova A, et al. Trastuzumab in combination with chemotherapy versus chemotherapy alone for treatment of HER2positive advanced gastric or gastro-oesophageal junction cancer (ToGA): a phase 3, open-label, randomised controlled trial[J]. Lancet. 2010, 376(9742): 687-697.

[46] Waddell T, Chau I, Cunningham D, et al. Epirubicin, oxaliplatin, and capecitabine with or without panitumumab for patients with previously untreated advanced oesophagogastric cancer (REAL3): a randomised, open-label phase 3 trial[J]. Lancet Oncol. 2013, 14(6): 481-489.

[47] Fuchs C S, Tomasek J, Yong C J, et al. Ramucirumab monotherapy for previously treated advanced gastric or gastro-oesophageal junction adenocarcinoma (REGARD): an international, randomised, multicentre, placebo-controlled, phase 3 trial[J]. Lancet. 2014, 383(9911): 31-39.

[48] Wilke H, Muro K, Van Cutsem E, et al. Ramucirumab plus paclitaxel versus placebo plus paclitaxel in patients with previously treated advanced gastric or gastro-oesophageal junction adenocarcinoma (RAINBOW): a double-blind, randomised phase 3 trial[J]. Lancet Oncol. 2014, 15(11): 12241235 .

[49] Nason G J, Tareen F, Mortell A. The top 100 cited articles in urology: An update[J]. Can Urol Assoc J. 2013, 7(1-2): E16-E24.

[50] Brandt J S, Downing A C, Howard D L, et al. Citation classics in obstetrics and gynecology: the 100 most frequently cited journal articles in the last 50 years[J]. Am J Obstet Gynecol. 2010, 203(4): 351-355.

\section{Figures}




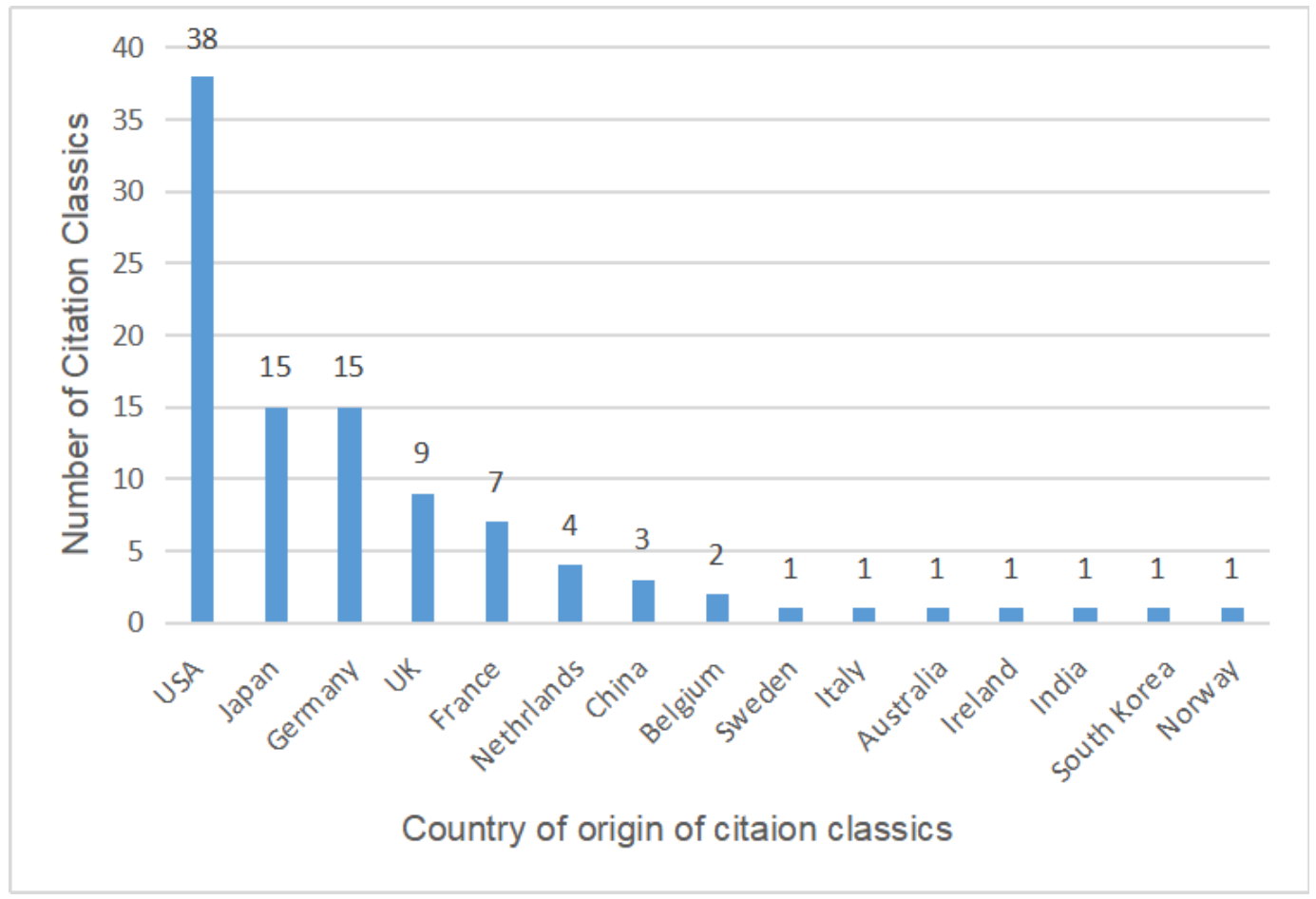

\section{Figure 1}

Countries of origin of the 100 most-cited articles.

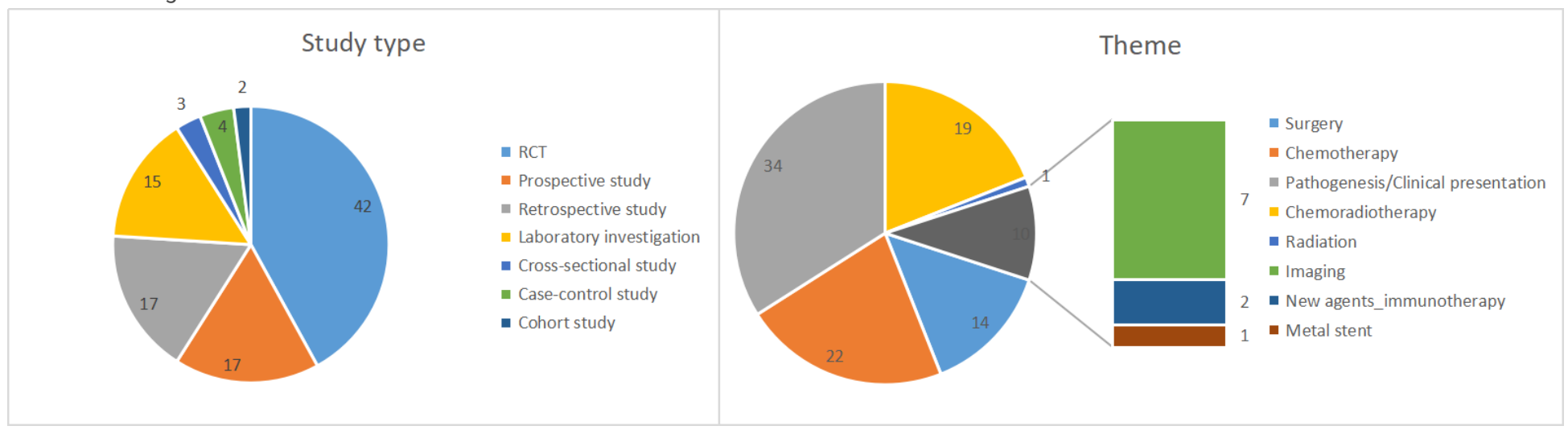

\section{Figure 2}

Distributions of the 100 most-cited articles by study design and theme. 


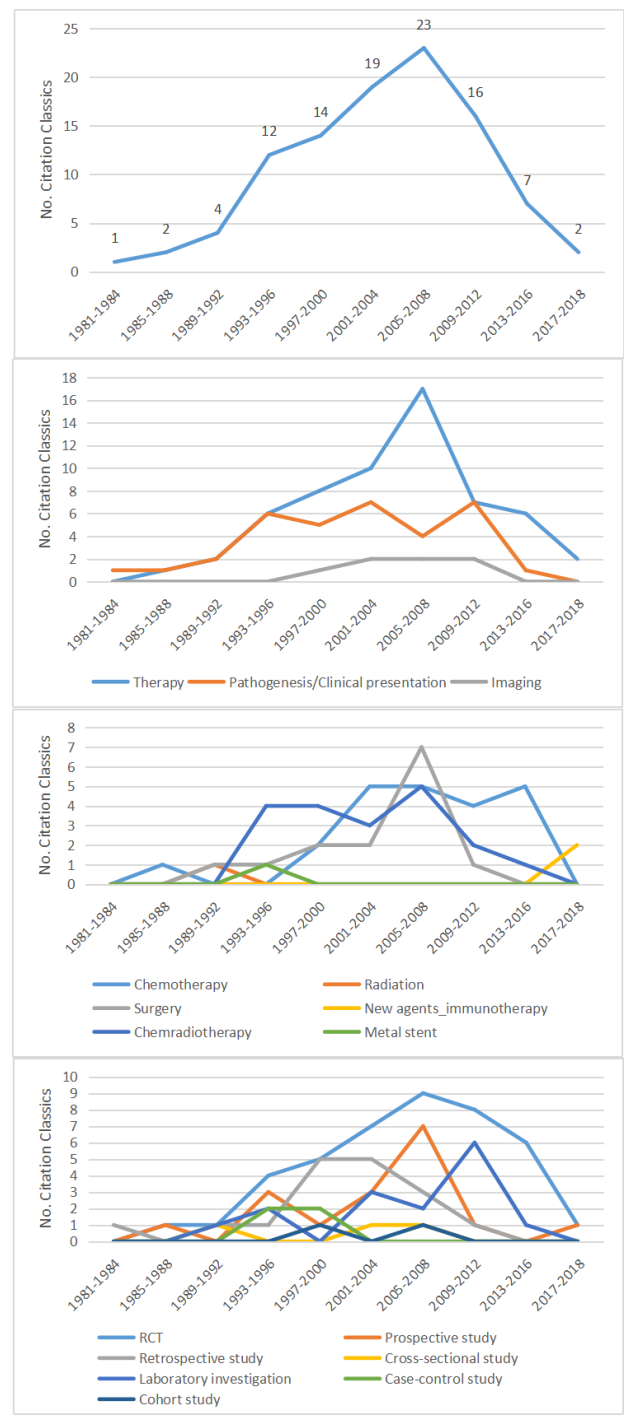

Figure 3

Trends in esophageal cancer reported in citation classics over time. (A) Total number of citation classics by epoch. (B) Trends in themes reported in citation classics by epoch. (C) Trends in therapies reported in citation classics by epoch. (D) Trends in study designs of citation classics by epoch. 
1997、1999、2002

RTOG8501/RTOG9405

Radical concurrent chemoradiotherapy for locally advanced esophageal cancer (AE/ESCC)

\section{8}

Fluoropyrimidine and oxaliplatin/cisplatin for metastatic GAC and AEGJ (first-line therapy)

\section{ToGA}

Trastuzumab in combination with chemotherapy for HER2-positive metastatic AEGJ and GAC

(first-line therapy)

\section{RAINBOW}

Ramucirumab plus paclitaxel for GAC and AEGJ (second-line therapy)

\section{ATTRACTION-3}

Nivolumab for esophageal squamous cell carcinoma (second-line therapy)

\section{INT0116}

Postoperative chemoradiotherapy

for $\mathrm{GAC}$ and $\mathrm{AEGJ}$

\section{FNCLCC/FFCD}

Perioperative chemotherapy for resectable $\mathrm{AE}, \mathrm{AEGJ}$ or GAC

\section{CROSS}

Preoperative Chemoradiotherapy for Esophageal or Junctional Cancer (AE/ESCC)

\section{NEOCRTEC5010}

Neoadjuvant chemoradiotherapy for locally advanced esophageal squamous cell carcinoma

\section{Figure 4}

Timeline of important historical advances in esophageal cancer. AE, adenocarcinoma of the esophagus; AEGJ, adenocarcinoma of the esophagogastric junction; GAC, gastric adenocarcinoma; ESCC, esophageal squamous cell carcinoma; HER2, human epidermal growth factor receptor 2. 